Draft version April 23, 2022

Preprint typeset using $\mathrm{LATEX}_{\mathrm{E}}$ style emulateapj v. 12/16/11

\title{
COMPARISON OF THE EXTRAPLANAR H $\alpha$ AND UV EMISSIONS IN THE HALOS OF NEARBY EDGE-ON SPIRAL GALAXIES
}

\author{
Young-Soo Jo ${ }^{1,2}$, Kwang-Il Seon ${ }^{1,3}$, Jong-Ho Shinn ${ }^{1}$, Yujin Yang ${ }^{1}$, Dukhang Lee ${ }^{1,4}$, And Kyoung-Wook Min ${ }^{2}$ \\ Draft version April 23, 2022
}

\begin{abstract}
We compare vertical profiles of the extraplanar $\mathrm{H} \alpha$ emission to those of the UV emission for 38 nearby edge-on late-type galaxies. It is found that detection of the "diffuse" extraplanar dust (eDust), traced by the vertically extended, scattered UV starlight, always coincides with the presence of the extraplanar $\mathrm{H} \alpha$ emission. A strong correlation between the scale heights of the extraplanar $\mathrm{H} \alpha$ and UV emissions is also found; the scale height at $\mathrm{H} \alpha$ is found to be $\sim 0.74$ of the scale height at FUV. Our results may indicate the multiphase nature of the diffuse ionized gas and dust in the galactic halos. The existence of eDust in galaxies where the extraplanar $\mathrm{H} \alpha$ emission is detected suggests that a larger portion of the extraplanar $\mathrm{H} \alpha$ emission than that predicted in previous studies may be caused by $\mathrm{H} \alpha$ photons that originate from H II regions in the galactic plane and are subsequently scattered by the eDust. This possibility raise a in studying the eDIG. We also find that the scale heights of the extraplanar emissions normalized to the galaxy size correlate well with the star formation rate surface density of the galaxies. The properties of eDust in our galaxies is on a continuation line of that found through previous observations of the extraplanar polycyclic aromatic hydrocarbons emission in more active galaxies known to have galactic winds.
\end{abstract}

Keywords: line: formation - radiative transfer - scattering - dust, extinction — ISM: structure — ISM: HII regions

\section{INTRODUCTION}

Study of the feedback between stars and interstellar medium (ISM) is essential to understand the formation and evolution of galaxies. Although the ISM in latetype galaxies including the Milky Way Galaxy are mostly concentrated in the galactic plane, it has been found that a considerable amount of materials exists at high altitudes above the midplane (e.g., Haffner et al. 2009, Putman et al. 2012; Seon et al. 2014, Hodges-Kluck \& Bregman 2014). The extraplanar material in the galactic halo traces infalling star-formation fuel and feedback from a galaxy's disk and is therefore a crucial component of galactic evolution that probes disk-halo interaction (Dettmar 2005; Putman et al. 2012).

Narrow band and spectroscopic observations of the $\mathrm{H} \alpha$ emission of nearby late-type edge-on galaxies have revealed the existence of extraplanar $\mathrm{H} \alpha$ emission from many galaxies with sufficient star formation rates (SFRs). The extraplanar $\mathrm{H} \alpha$ emission is generally believed to originate from the extraplanar diffuse ionized gas (hereafter, eDIG) (Rand 1996, Rossa \& Dettmar 2000, 2003a b, Rossa et al. 2004; Miller \& Veilleux 2003a b. Ho et al. 2016). The eDIG is believed to be maintained by photoionization by ionizing photons (Lyman continuum; Lyc) that are mainly produced by Otype stars in the galactic plane (Reynolds 1984, Domgorgen \& Mathis 1994, Ferguson et al. 1996; Zurita et al.

\footnotetext{
${ }^{1}$ Korea Astronomy and Space Science Institute (KASI), 776 Daedeokdae-ro, Yuseong-gu, Daejeon, 34055, Korea; stspeak@gmail.com

2 Korea Advanced Institute of Science and Technology (KAIST), 291, Daehak-ro, Yuseong-gu, Daejeon, 34141, Korea

${ }^{3}$ Astronomy and Space Science Major, Korea University of Science and Technology, Daejeon, 34113, Korea

${ }^{4}$ York University, 4700 Keele Street, Toronto, Ontario, M3J 1P3, Canada
}

2002; Wood \& Mathis 2004, Haffner et al. 2009, Barnes et al. 2015).

However, it is still not clear whether Lyc leaked out of $\mathrm{H}$ II regions in the galactic plane is the major ionization source of the high latitude and interarm gas (e.g., Seon 2009; Seon et al. 2011; Dong \& Draine 2011; FloresFajardo et al. 2011; Jones et al. 2017). Seon (2009) pointed out that an incredibly low absorption coefficient of Lyc is required to explain the diffuse $\mathrm{H} \alpha$ emission of a face-on galaxy M 51 with the standard photoionization model. One alternative explanation of the extraplanar $\mathrm{H} \alpha$ emission was proposed by Dong \& Draine (2011). The diffuse $\mathrm{H} \alpha$ emission in their model originates from gas that was photoionized in the past, but which is currently cooling and recombining; the ionizing radiation should last only for a very short time $\left(\sim 10^{5} \mathrm{yrs}\right)$, compared to $O$ star lifetimes $\left(\sim 3 \times 10^{6} \mathrm{yrs}\right)$, before the photoionization switches off and the gas begins to cool. Therefore, the ionizing radiation should be mostly provided by runaway $\mathrm{OB}$ stars with velocities of $\gtrsim 100 \mathrm{~km}$ $\mathrm{s}^{-1}$.

The processes that expel gas from the galactic disk may also act on the interstellar dust grains. It has been suggested that dust could be elevated by radiation pressure from the galactic disk into the halo (Greenberg et al. 1987; Franco et al. 1991; Ferrara et al. 1991) and/or by hydrodynamic motions due to supernovae and stellar winds (Howk \& Savage 1997). The extraplanar dust (hereafter, eDust) in galactic halos (or thick disks) can be utilized to study the feedback process in the cold phase of the ISM. High-resolution optical images of nearby edge-on galaxies have revealed an extensive filamentary structure of the eDust seen in absorption against the background stellar light of the bulge and thick stellar disk (Howk \& Savage 1997, 1999, 2000, Thompson et al. 
2004). Many studies in the mid-infrared (MIR) and farIR (FIR) wavelengths have provided evidence of eDust (Alton et al. 2000a b, Irwin \& Madden 2006, Burgdorf et al. 2007; Kaneda et al. 2009, Verstappen et al. 2013 Bocchio et al. 2016). Far-ultraviolet (FUV) and/or nearUV (NUV) observations of edge-on galaxies have revealed the stellar continuum scattered into the line of sight by the eDust (Seon et al. 2014, Hodges-Kluck \& Bregman 2014 Shinn \& Seon 2015; Hodges-Kluck et al. 2016). The FUV emission in the galactic outflows of starburst galaxies were also attributed to starlight scattered by dust in the outflow (Hoopes et al. 2005). The UV continuum emission mostly originates from OB stars in the galactic thin disk and hence the UV reflection halo caused by the eDust can be used to estimate the amount of eDust. Based on this idea, Seon et al. (2014) and Shinn \& Seon (2015) quantitatively derived the amount of eDust by comparing dust radiative transfer models with the observed UV images of edge-on galaxies. Baes \& Viaene (2016) showed that the spectral energy density of NGC 3628 is well reproduced using the dust and stellar geometries obtained by Shinn \& Seon (2015).

Rossa \& Dettmar (2003b) found a correlation between the presence/non-presence of eDust and eDIG in spiral galaxies; absorbing dusty features at high altitudes are usually found in the galaxies where the eDIG is also detected. The simultaneous existence of the eDust and eDIG indicates the multiphase nature of the ISM in galactic halos. In this regard, Howk \& Savage (2000) and Rueff et al. (2013) compared the absorbing, filamentary dust structures in high-resolution optical ( $B V I$ bands) images to the $\mathrm{H} \alpha$ emission of edge-on galaxies, but found that the filamentary morphologies of the dust absorption have no counterpart in the smoothly distributed $\mathrm{H} \alpha$ emission. They concluded that the diffuse eDIG and filamentary eDust trace physically distinct phases of the thick disk ISM. However, it should be noted that the absorbing dust features trace only opaque clouds with optical depths of $\gtrsim 1$, as noted in Seon et al. (2014), and the $\mathrm{H} \alpha$ emission would be extinguished by the absorbing dust clouds. Thus, the absence of a spatial correspondence between the absorbing dust and $\mathrm{H} \alpha$ emission does not necessarily imply distinct phases. The total amount of eDust, which is missing in the high-resolution optical studies, is better traced by observing the scattered starlight in UV wavelengths, as studied by Seon et al. (2014) and Shinn \& Seon (2015). Therefore, the multiphase nature of eDust and eDIG can be best studied by comparing the UV halo and the extraplanar $\mathrm{H} \alpha$ emission.

Moreover, we note that the diffuse eDust that scatters the UV starlight from the midplane (Hodges-Kluck \& Bregman 2014, Seon et al. 2014, Shinn \& Seon 2015; Hodges-Kluck et al. 2016) and produces the UV reflection halo has a potential to scatter the $\mathrm{H} \alpha$ photons originating from H II regions in the galactic plane. Ferrara et al. (1996) investigated the amount of $\mathrm{H} \alpha$ photons that originates in $\mathrm{H}$ II regions and scattered by dust at high altitude and found that $\sim 10 \%$ of the extraplanar $\mathrm{H} \alpha$ emission at $z \sim 600 \mathrm{pc}$ can be attributed to the scattered light (see also Wood \& Reynolds (1999) for a similar model in the Milky Way Galaxy). However, they took into account only a thin dust disk with a scale height of $\sim 0.2 \mathrm{kpc}$ to calculate the fraction of the scattered $\mathrm{H} \alpha$ component. The presence of eDust in galaxies where the extraplanar $\mathrm{H} \alpha$ emission is detected will raise the fraction of scattered $\mathrm{H} \alpha$ photons compared to the estimation of Ferrara et al. (1996) and thus decrease the amount of in-situ photoionized gas in the halo.

The above two concerns regarding the multiphase nature of the extraplanar ISM and the possibility of $\mathrm{H} \alpha$ to be scattered by the eDust motivated the present study of comparing the extraplanar $\mathrm{H} \alpha$ and UV emissions in the halos of nearby edge-on late-type galaxies. In this study, we compare the vertical profiles of $\mathrm{H} \alpha$ emission to those of UV emission in the nearby edge-on galaxies. In Section 2, we describe the sample galaxies. We examine correlation relations between the vertical profiles of the extraplanar $\mathrm{H} \alpha$ and UV emissions in Section 3. Correlation of the extraplanar emissions with the star formation rate (SFR) is also investigated. Section 4 presents a summary and discussion.

\section{DATA}

We analyzed the narrow band $\mathrm{H} \alpha$ and UV images of the nearby late-type edge-on galaxies which were taken from previous $\mathrm{H} \alpha$ and UV galaxy surveys. The $\mathrm{H} \alpha$ images were obtained from the following databases: the Spitzer Local Volume Legacy (LVL; Kennicutt et al. 2008 Dale et al. 2009) survey, the Spitzer Infrared Nearby Galaxies Survey (SINGS; Kennicutt et al. 2003. Moustakas et al. 2010), the Survey for Ionization in Neutral Gas Galaxies (SINGG; Meurer et al. 2006), the $\mathrm{H} \alpha$ Galaxy Survey (H $\alpha$ GS; James et al. 2004), the H $\alpha$ narrow band imaging survey of galaxies $(\mathrm{H} \alpha 3$; Gavazzi et al. 2003, 2012), and the $\mathrm{H} \alpha$ survey (henceforth Rossa) of Rossa \& Dettmar (2003a b). The $\mathrm{H} \alpha$ images are available in the relevant websites of LVI 5 , SINGS6 6 , SINGG7 $\mathrm{H} \alpha \mathrm{GS}, \mathrm{H} \alpha 3^{9}$, and Ross: ${ }^{10}$. In order to compare the $\mathrm{H} \alpha$ images with the FUV and NUV images, we also have retrieved the GALEX archival data ${ }^{11}$ (Galaxy Evolution Explorer; Martin et al. 2005, Morrissey et al. 2005, 2007) of the sample galaxies. In total, 38 edgeon late-type galaxies with a distance of less than $30 \mathrm{Mpc}$ were selected. Visual inspection was performed to exclude galaxies with noticeable spiral or asymmetry patterns. The data with the highest signal-to-noise ratio were adopted if the galaxy was observed several times in different databases.

Table 1 shows the sample galaxies and their basic information mostly taken from the NASA/IPAC Extragalactic Database (NED) ${ }^{12}$ The $\mathrm{H} \alpha$ data for 12 galaxies were obtained from LVL (Kennicutt et al. 2008: Dale et al. 2009), 12 galaxies from SINGG (Meurer et al. 2006), 2 galaxies from SINGS(Kennicutt et al. 2003; Moustakas et al. 2010), 4 galaxies from $\mathrm{H} \alpha \mathrm{GS}$ (James et al. 2004), 6 galaxies from $\mathrm{H} \alpha 3$ (Gavazzi et al. 2003, 2012), and 2 galaxies from Rossa (Rossa \& Dettmar 2003a b).

The entries in Table 1 are organized as follows: column (1) - the running index number, column (2) - galaxy

\footnotetext{
5 http://irsa.ipac.caltech.edu/data/SPITZER/LVL/summary.html

6 http://irsa.ipac.caltech.edu/data/SPITZER/SINGS/

7 http://sungg.pha.jhu.edu/PubData/Portal/index.html

8 http://www.astro.ljmu.ac.uk/HaGS/tits/index.html

9 http://goldmine.mib.intn.it/

16 https://ned.ipac.caltech.edu/cgi-

bin $/$ objsearch?refcode $=2003 \mathrm{~A} \% 26 \mathrm{~A} \ldots .406 . .505 \mathrm{~K} \&$ search_type $=$ Search

ii http://galex.stsci.edu/GR6/

12 https://ned.ipac.caltech.edu/
} 
name taken from the NED's preferred object name, column (3) - galaxy morphology, column (4) - galactic longitude, column (5) - galactic latitude, column (6) - average value of the redshift-independent distances taken from NED, column (7) - major axis diameter in arcmin, column (8) - major axis diameter in kpc, column (9) - integrated $\mathrm{H} \alpha$ luminosity taken from the reference papers, column (10) - star formation rate $\mathrm{SFR}_{\mathrm{H} \alpha}$ estimated using the $\mathrm{H} \alpha$ luminosity (column 9), column (11) - star formation rate $\mathrm{SFR}_{\mathrm{FIR}}$ estimated using the far-infrared (FIR) luminosity $L_{\mathrm{FIR}}$, and column (12) - references for the $\mathrm{H} \alpha$ data. The star formation rates, shown in columns (10) and (11), were estimated using the following relations (Kennicutt 1998):

$$
\begin{aligned}
\mathrm{SFR}_{\mathrm{H} \alpha} & =\frac{L_{\mathrm{H} \alpha}}{1.26 \times 10^{41} \mathrm{erg} \mathrm{s}^{-1}} M_{\odot} \mathrm{yr}^{-1} \\
\mathrm{SFR}_{\mathrm{FIR}} & =\frac{L_{\mathrm{FIR}}}{2.2 \times 10^{43} \mathrm{erg} \mathrm{s}^{-1}} M_{\odot} \mathrm{yr}^{-1}
\end{aligned}
$$

where the FIR luminosity $L_{\mathrm{FIR}}$ was calculated using the relation of Rice et al. (1988), $L_{\mathrm{FIR}}=1.51 \times$ $10^{39} d_{\mathrm{Mpc}}^{2}\left(2.58 f_{60}+f_{100}\right)$ erg $\mathrm{s}^{-1}$. Here, $f_{60}$ and $f_{100}$ are the fluxes at 60 and $100 \mu \mathrm{m}$, respectively, in Jy obtained from the IRAS catalog (Moshir \& al 1990). The FIR luminosities for seven galaxies (UGCA 442, UGCA 193, ESO 347-G 017, IC 4951, UGC 08313, NGC 5229, and NGC 5023) that were not provided in the IRAS catalog were calculated using the monochromatic luminosity $L_{\mathrm{FIR}}=4 \pi d^{2}(c / \lambda) f_{70}=5.39 \times 10^{39} d_{\mathrm{Mpc}}^{2} f_{70} \mathrm{erg}$ $\mathrm{s}^{-1}$, where $f_{70}$ is the flux at $70 \mu \mathrm{m}$ in Jy obtained from the MIPS catalog (Multiband Imaging Photometer for Spitzer; Dale et al. 2009). The SFR FIR $_{\text {for three galaxies }}$ (NGC 7412A, ESO 249-G 035, NGC 3365) that have no FIR luminosity were estimated from the empirical relation $\mathrm{SFR}_{\mathrm{FIR}}=1.31 \times \mathrm{SFR}_{\mathrm{H} \alpha}^{1.54}$. This relation was derived by using the correlation between $\mathrm{SFR}_{\mathrm{FIR}}$ and $\mathrm{SFR}_{\mathrm{H} \alpha}$ of our galaxies, of which the FIR luminosities are available. Thus, the relation would be suitable for edge-on galaxies in a statistical sense, although $\mathrm{H} \alpha$ emission is not a good tracer of SFR for edge-on galaxies.

The $\mathrm{H} \alpha$ and UV images of 38 galaxies were processed in the following order. First, point-like sources as well as extended sources except the target galaxy were masked out using Source Extractor (Bertin \& Arnouts 1996). Most point sources in the $\mathrm{H} \alpha$ images were removed through the continuum subtraction process. Second, the masked image was rotated about the center of the galaxy to align the major axis of the galaxy with the horizontal axis of the image. Third, the rotated image was cropped to a rectangular shape, putting the center of the galaxy at the center of the rectangle. The final images and vertical profiles in the $\mathrm{H} \alpha, \mathrm{FUV}$, and NUV wavelengths for the 38 galaxies are shown in Figure 1 .

An important factor affecting vertical profiles of the extraplanar emission is an extended wing of the point spread function (PSF) of a telescope (Sandin 2014, 2015). Shinn \& Seon (2015) took this effect into account in dust radiative transfer models and Hodges-Kluck et al. (2016) subtracted the contamination by the PSF-wing from the observed images. The effect of the extended wing is severe only when studying surface brightnesses that are much lower than $\sim 10^{-2}(\sim 5 \mathrm{mag})$ of peak intensity at the galactic plane. As can be seen in Figure 1. we mainly focus on higher intensity levels. To test the PSFwing effect, we examined whether the scale heights of extraplanar $\mathrm{H} \alpha, \mathrm{FUV}$, and NUV emissions, derived in Section 3.2, systematically increase with distance to the galaxies. But, no systematic trend was found. Therefore, we conclude that this effect is not significant for galaxies in our sample. The contamination by the extended wing of PSF may marginally change the scale heights of the extraplanar emission measured in the present study. However, this effect does not significantly alter the results presented in this paper.

We also note that, even at small deviations from $90^{\circ}$, the projected disk may appear as vertical emission. Some of the galaxies in Figure 1 do not appear to be completely edge-on (e.g., NGC 5951, NGC 493, NGC 803, NGC 4020, NGC 3365, IC 2000, and NGC 5356). In addition, some of the galaxies show disturbed disks in optical and Near-IR images (e.g., NGC 5107, NGC 4631, NGC 3432, and NGC 3628). Therefore, in Figures 2 to 5. we used different colors to denote these galaxies to see if they stand out in any particular way: blue for the less-inclined galaxies and yellow for the disturbed disks. In the figures, it can be immediately recognized that our results are not affected by the galaxies.

\section{RESULTS}

\subsection{Morphology}

As shown in Figure 1 , the morphology of the $\mathrm{H} \alpha$ emission, especially in the galactic plane, is in general more compact than those shown in the FUV and NUV images, although deeper and higher-resolution observations are required for a more detailed comparison. In other words, there is less contrast in the UV light, compared to the $\mathrm{H} \alpha$ emission. We also note that the radial and vertical extents appear to be smaller at $\mathrm{H} \alpha$.

The trend is consistent with the anti-correlation between the FUV to $\mathrm{H} \alpha$ intensity ratio and $\mathrm{H} \alpha$ intensity found in 10 face-on spiral galaxies (Hoopes \& Walterbos 2000, Hoopes et al. 2001) and two starburst galaxies (Hoopes et al. 2005). A similar trend was also found in the Milky Way Galaxy (Seon et al. 2011). Moving from a bright region into diffuse regions, the FUV to $\mathrm{H} \alpha$ intensity ratio increases and thus the $\mathrm{H} \alpha$ intensity decreases faster than the decrease at FUV. This property is equivalent to the more compact morphology at $\mathrm{H} \alpha$. This is due to the fact that $\mathrm{H}$ II regions are more spatially clumped than stars that emit the FUV and NUV continuum. The $\mathrm{H} \alpha$ emission originates mainly from the $\mathrm{H}$ II regions around $\mathrm{OB}$ associations, while the FUV and NUV emissions arise not only from OB associations but also from late field $\mathrm{OB}$ and A stars, which are more spatially extended than OB associations. Further discussion of the morphology is given in Section 4 .

\subsection{Vertical Profile}

The vertical profiles of the $\mathrm{H} \alpha, \mathrm{FUV}$, and NUV emissions for the 38 edge-on galaxies were obtained by horizontally averaging each image and then the profiles, denoted by black solid lines in Figure 1, were fitted with an exponential function. The adopted exponential function 
to fit the extraplanar emission is

$$
y=a_{0} \exp \left(-\frac{\left|x-a_{1}\right|}{a_{2}}\right)+a_{3}+a_{4} x,
$$

where the first term on the right-hand side is an exponential function representing the vertical profile of the extraplanar emission and the remaining terms are a linear function representing the background of the profile. The parameters of the exponential function in Equation (3) are the scale height $\left(a_{2}\right)$, the peak intensity $\left(a_{0}\right)$, and the location of the galactic center $\left(a_{1}\right)$, respectively. The resulting best-fit exponential functions are represented by red dashed lines in Figure 1. The background levels are denoted by red dotted lines in Figure 11. The scale height found for each image is shown at the top left corner of each figure together with its $1 \sigma$ error in units of kpc. The best-fit parameters are shown in Table 2 .

The thin disk component of dust with a scale height of $\sim 0.2 \mathrm{kpc}$ was assumed to be mostly confined in a region with intensity brighter than a certain threshold marked by blue dotted lines in Figure 1. The region above the threshold level was excluded from the fit to minimize contamination by the thin disk component in estimating the extraplanar component. The threshold for most galaxies was set to be $e^{-2}$ times the difference between the peak intensity and the background. For 10 galaxies (ESO 249-G 035, NGC 7412A, NGC 1311, NGC 0784, UGC 08313, IC 2233, NGC 3510, NGC 4313, IC 5176, and NGC 3628) that have relatively poor signal-to-noise ratios, we set the threshold as $e^{-1}$ times the difference. The threshold was empirically chosen to discriminate the extraplanar component from the thin disk component. It should be noted that the radial scale length of the thin dust disk tends to be larger than that of the stellar disk (Xilouris et al. 1997, 1998; De Geyter et al. 2014, Seon et al. 2014 Shinn \& Seon 2015). Therefore, the observed peak intensity is an attenuated value by the thin dust disk and the adopted threshold level is much lower than the value estimated by multiplying $e^{-2}$ or $e^{-1}$ to the intrinsic peak intensity. This implies that we are analyzing regions far enough from the galactic plane. The bestfit scale height would be higher than the value expected from the galactic thin disk, if there is an additional, thick component in the halo as detected in Seon et al. (2014), Shinn \& Seon (2015), Hodges-Kluck \& Bregman (2014), and Hodges-Kluck et al. (2016). We also adopted lower thresholds corresponding to more outer regions from the midplane and repeated the analysis, but the following results were not significantly altered.

We compare the scale heights estimated from the $\mathrm{H} \alpha$, FUV, and NUV data in Figure 2. Figure 2(a) shows a strong correlation between the scale heights of the FUV $\left(Z_{\mathrm{FUV}}\right)$ and NUV $\left(Z_{\mathrm{NUV}}\right)$ emissions. The scale height of the $\mathrm{H} \alpha$ emission $\left(\mathrm{Z}_{\mathrm{H} \alpha}\right)$ is compared to that of the FUV emission $\left(\mathrm{Z}_{\mathrm{FUV}}\right)$ in Figure 2(c). In Figures 2(b) and $(d)$, the scale heights normalized to the size of major axis $\left(\mathrm{D}_{25}\right)$ are compared. The black dashed diagonal lines in Figure 2 indicate one-to-one correspondence lines. There are strong correlations not only between $\mathrm{Z}_{\mathrm{FUV}}$ and $\mathrm{Z}_{\mathrm{NUV}}$ but also between the normalized values $\mathrm{Z}_{\mathrm{FUV}} / D_{25}$ and $\mathrm{Z}_{\mathrm{NUV}} / D_{25}$. The correlation coefficient between $\mathrm{Z}_{\mathrm{FUV}}$ and $\mathrm{Z}_{\mathrm{NUV}}\left(\mathrm{Z}_{\mathrm{FUV}} / \mathrm{D}_{25}\right.$ and $\left.\mathrm{Z}_{\mathrm{NUV}} / \mathrm{D}_{25}\right)$ is obtained as 0.96 (0.88) in Figure 2(a) (Figure 2(b)). In
Figure 2(c) (Figure 2(d)), the correlation coefficient between $\mathrm{Z}_{\mathrm{H} \alpha}$ and $\mathrm{Z}_{\mathrm{FUV}}\left(\mathrm{Z}_{\mathrm{H} \alpha} / \mathrm{D}_{25}\right.$ and $\left.\mathrm{Z}_{\mathrm{FUV}} / \mathrm{D}_{25}\right)$ is found to be 0.89 (0.67). The correlations shown in Figures 2 (c) and (d) are less significant than the cases of $\mathrm{Z}_{\mathrm{FUV}}$ and $\mathrm{Z}_{\mathrm{NUV}}$, but still strong. In Figures 2(a) (Figure 2(b)), the blue dot-dashed line denotes the linear line representing a direct proportional relation between $\mathrm{Z}_{\mathrm{FUV}}$ and $\mathrm{Z}_{\mathrm{NUV}}$ $\left(\mathrm{Z}_{\mathrm{FUV}} / \mathrm{D}_{25}\right.$ and $\left.\mathrm{Z}_{\mathrm{NUV}} / \mathrm{D}_{25}\right)$. The proportional relations are also shown in the figures. As shown in the figure, the scale height of the NUV emission is found to be in general the same as (but slightly lower than) that of the FUV emission. The scale height of the $\mathrm{H} \alpha$ emission tends to be smaller than that of the FUV emission. In Figures 2 (c) and (d), the blue triple-dot-dashed lines denote the Tines corresponding to $Z_{\mathrm{H} \alpha}=0.5 Z_{\mathrm{FUV}}$. The $\mathrm{H} \alpha$ intensity scales with the emission measure (defined as the square of the number density of electrons integrated over the volume of ionized gas); the scale height of electron (ionized gas) may be twice as large as the $\mathrm{H} \alpha$ scale heights. If the ionized gas traced by $\mathrm{H} \alpha$ emission is well mixed with dust, then the $\mathrm{H} \alpha$ scale height will be half of the dust scale height.

The sample galaxies were divided into two groups for convenience, as shown in Table 2. Group A with a scale height less than $0.4 \mathrm{kpc}$ in both UV wavelength bands and Group B with a scale height greater than $0.4 \mathrm{kpc}$ in both UV bands. The scale height threshold dividing Groups A and B was determined based on the observations that the scale height of $\mathrm{OB}$ stars that are the main source of the UV continuum is $\lesssim 0.2 \mathrm{kpc}$ and the scale height of thin dust disk tends to be $\sim 0.2 \mathrm{kpc}$ (ranging from $\sim 0.1 \mathrm{kpc}$ to $\sim 0.4 \mathrm{kpc}$ ) (Xilouris et al. 1997, 1998, 1999, Alton et al. 2004, De Geyter et al. 2014). The galaxies that have relatively small inclinations or show disturbed disks in optical images belong to Group B. In Figures 2 to 5 , Groups $\mathrm{A}$ is denoted by black diamonds. Group B is denoted by red, blue, and yellow squares. The blue and yellow squares indicate the lessinclined galaxies and disturbed disks, respectively. The red squares denote remaining galaxies in Group B. The black dashed, vertical, and horizontal lines in Figures 2 (a) and (c) indicate lines corresponding to $\mathrm{Z}_{\mathrm{FUV}}=0.4$ $\mathrm{kpc}$ and $\mathrm{Z}_{\mathrm{NUV}}=0.4 \mathrm{kpc}$, respectively. The symbol size in Figures 2 to 5 is proportional to the logarithm of the galaxy size $\left(\log \mathrm{D}_{25}\right)$.

The average scale heights at $\mathrm{H} \alpha$ and FUV for Group $\mathrm{A}$ are $\mathrm{Z}_{\mathrm{H} \alpha}=0.21 \pm 0.5 \mathrm{kpc}$ and $\mathrm{Z}_{\mathrm{FUV}}=0.23 \pm 0.6$ $\mathrm{kpc}$, respectively. These values are consistent with the scale height of the thin dust disk as well as OB associations. This indicates that the Group A galaxies have no (or negligible) additional geometrically thick dust component and we are detecting the exponential tail of the thin disk. On the other hand, the larger scale heights found in Group B imply the presence of an additional component in the galaxies of Group B. The scale height $\mathrm{Z}_{\mathrm{H} \alpha}$ of Group A ranges from $\sim 0.1$ to $0.3 \mathrm{kpc}$ except IC 5052. Most galaxies in Group B, except seven galaxies, have a scale height at $\mathrm{H} \alpha$ larger than $0.4 \mathrm{kpc}$. We also note that the galaxies with small $Z_{\mathrm{H} \alpha}(<0.4 \mathrm{kpc})$ in Group B have relatively small $\mathrm{Z}_{\mathrm{FUV}}$. In other words, the galaxies with the additional extraplanar $\mathrm{H} \alpha$ emission appear to have the extraplanar FUV emission as well. The trend is consistent with the results of Howk \& Savage (2000) and Rossa \& Dettmar (2003b), in that they also 
found a similar trend by comparing the clumpy features of eDust and the $\mathrm{H} \alpha$ emission. However, it should be noted that $Z_{\mathrm{H} \alpha}$ is in general smaller than $Z_{\mathrm{FUV}}$. The average ratio of $Z_{\mathrm{H} \alpha}$ to $Z_{\mathrm{FUV}}$ is $0.74 \pm 0.30$ for the galaxies in Group B. This point will be discussed in Section 4.

The normalized scale heights $\left(\mathrm{Z}_{\mathrm{FUV}} / \mathrm{D}_{25}\right.$ and $\left.\mathrm{Z}_{\mathrm{H} \alpha} / \mathrm{D}_{25}\right)$ in Figure 2(b) and (d) range from 0.01 to 0.1 . In the figure, averages of the normalized scale heights of Group B appear to be slightly higher than those of Group A, although the differences are not large. The absence of a substantial difference in the normalized scale height between the two groups suggests that the scale height tends to increase with the galaxy size. Nonetheless, the finding that the normalized scale height does not approach to a single value indicates that the scale height depends on other properties (e.g., star formation rate) of galaxies as well. This issue will be discussed in the next section.

\subsection{Comparison with Star Formation Rate}

Most phenomena in spiral galaxies are closely associated with the star formation activity. We therefore compare the scale heights of FUV and $\mathrm{H} \alpha$ emissions with the star formation rates derived from the FIR luminosity $\left(\mathrm{SFR}_{\mathrm{FIR}}\right)$ of host galaxies in Figure 3 . It is clear that both the scale heights $\mathrm{Z}_{\mathrm{FUV}}$ and $\mathrm{Z}_{\mathrm{H} \alpha}$ strongly correlate

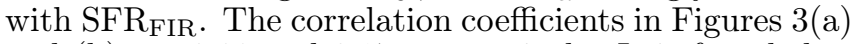
and (b) are 0.92 and 0.87 , respectively. It is found that the scale heights are well described by a power law function of SFR FIR. The best-fit power law function is overplotted as a black dashed line in the figure. The equation describing the best-fit power law is also shown at the top left corner. In Figure 3, the condition for the detection of the extraplanar emission is $\mathrm{SFR}_{\mathrm{FIR}} \gtrsim 0.03 M_{\odot} \mathrm{yr}^{-1}$.

For a given SFR, the scale height tends to increase with the galaxy size. For instance, the three galaxies NGC 5107, NGC 4313, and NGC 3365 with SFR $\sim 0.1 M_{\odot}$ $\mathrm{yr}^{-1}$ show the trend clearly. Figure 4 shows a strong correlation between the scale height and the galaxy size, although the correlation is slightly weaker than the correlation between the scale height and SFR shown in Figure 3 Figure 4 also shows that the scale height increases with SFR for a given galaxy size, as in Figures 2 and 3 . The stronger correlation of the scale height with SFR than with the galaxy size is ascribed to the wider dynamic range of SFR.

Figures 5(a) and (b) compare the scale heights normalized by $\mathrm{D}_{25}$ with the surface density of SFR $\left(\Sigma_{\mathrm{SFR}, \mathrm{FIR}} \equiv\right.$ $\mathrm{SFR}_{\mathrm{FIR}} / \pi D_{25}^{2}$ ) of the host galaxies. The (nonnormalized) scale heights are compared with the surface density of SFR in Figures 5(c) and (d). Both the normalized and non-normalized scale heights of the FUV and $\mathrm{H} \alpha$ emissions show a good correlation with $\Sigma_{\mathrm{SFR} \text {,FIR }}$. The correlation of the SFR surface density is stronger with the $\mathrm{H} \alpha$ scale height than with the FUV scale height. It is interesting that the normalized scale height of Group A appears to decrease as the galaxy size, indicated by the symbol size, increases. On the other hands, there is no clear tendency between the normalized scale height and the galaxy size for the Group B galaxies.

McCormick et al. (2013) found a correlation between the SFR surface density and the normalized scale height of the extraplanar emission by polycyclic aromatic hydrocarbons (PAHs) for 16 local, active galaxies known to have galactic winds. Shinn \& Seon (2015) modeled the extraplanar dust of six nearby galaxies using a radiative transfer simulation for FUV images, and compared the obtained scale height of eDust with the SFR surface density for three targets (NGC 891, NGC 3628, and UGC 11794), which apparently show the extraplanar FUV emission. Since the galaxies analyzed in McCormick et al. (2013) are more active than the galaxies in this paper, it would be interesting to combine their results with ours. Figure 6 shows the SFR surface density as a function of the normalized scale height for our galaxies in Group B together with the results of McCormick et al. (2013) and Shinn \& Seon (2015). The blue pluses denote 16 galaxies of McCormick et al. (2013) and the black asterisks indicate three galaxies of Shinn \& Seon (2015). The red triangles and orange crosses indicate the results obtained from the extraplanar FUV and $\mathrm{H} \alpha$ emissions, respectively, of 21 galaxies (Group B galaxies) in the present study. Note that two of the galaxies (NGC 891 and NGC 3628) in Shinn \& Seon (2015) are also included in the present study. It is clear that the correlation relation between the normalized scale height of the $\mathrm{PAH}$ emission and the SFR surface density is consistent with the relation estimated using the extraplanar FUV and $\mathrm{H} \alpha$ emissions. This implies that the correlation relation between the normalized scale height and the SFR surface density holds for a very wide range of SF activity.

\section{SUMMARY AND DISCUSSION}

We measured the vertical scale heights of the extraplanar $\mathrm{H} \alpha, \mathrm{FUV}$, and NUV emissions for 38 nearby edge-on galaxies. The scale height at NUV is found to be similar to or slightly higher than the scale height at FUV. This might be due to the fact that the NUV continuum source could be a slightly later-type and thus has a slightly higher scale height than the source of the FUV continuum. It is also found that galaxies with the extraplanar $\mathrm{H} \alpha$ emission always show the extraplanar FUV emission as well. The scale height of the $\mathrm{H} \alpha$ emission strongly correlates with the scale height of the UV emission. The scale height at $\mathrm{H} \alpha$ is found to be in general lower than that at UV.

Rossa \& Dettmar (2003b) found a correlation between the presence/non-presence of eDust and eDIG in edge-on galaxies. Howk \& Savage (2000) and Rueff et al. (2013) found no spatial correspondence between the smoothly distributed $\mathrm{H} \alpha$ emission and the opaque eDust filamentary structure. However, it should be noted that these studies are based on the observations of opaque dust clumps at high altitudes. The present study has an advantage over these studies in that the UV reflection halos can probe the diffuse eDust, which was not detectable in the studies of Rossa \& Dettmar (2003b), Howk \& Savage (2000), and Rueff et al. (2013).

The scale heights of the extraplanar UV and $\mathrm{H} \alpha$ emissions are found to correlate with the star formation rate $\left(\mathrm{SFR}_{\mathrm{FIR}}\right)$, the galaxy size $\left(\mathrm{D}_{25}\right)$, and the SFR surface density $\left(\Sigma_{\text {SFR,FIR }}\right)$. The scale heights at the extraplanar emissions correlate more strongly with the SFR than with the size of galaxy. The SFR surface density correlates with the normalized scale heights measured at UV and $\mathrm{H} \alpha$. This result is in good agreement with the relation found for other galaxies in which the extraplanar PAH emission was detected by McCormick et al. (2013). 
We note that the galaxies observed in McCormick et al. (2013) are known to have galactic winds and thus are more active than our galaxies. The correlation suggests that the extraplanar ISM is closely associated with the galactic SF activities, such as stellar radiation pressure and supernovae feedback. The validity of the single correlation relation over a wide type of galaxies, ranging from normal star forming galaxies with no apparent galactic winds to starburst galaxies with strong winds, indicates that the properties of the extraplanar ISM do not abruptly change at a critical level as the SF activity increases.

The strong correlation between the presence/nonpresence of the FUV and $\mathrm{H} \alpha$ emissions and between their vertical profiles suggests two possibilities for the origin of the diffuse $\mathrm{H} \alpha$ emission. First, the extraplanar FUV and $\mathrm{H} \alpha$ emissions trace the eDust and ionized gas (eDIG), respectively, and the correlation is caused by the multiphase nature of the ISM in galactic halo. The eDIG in the traditional scenario is believed to be produced by ionizing photons transported through transparent pathways carved out by superbubbles or chimneys (Mac Low \& Ferrara 1999, Cecil et al. 2002; Strickland et al. 2004a b; Veilleux et al. 2005). Second, a substantial portion of the extraplanar $\mathrm{H} \alpha$ emission is caused by dust scattering of the photons originating from $\mathrm{H}$ II regions in the galactic disk.

Detailed model calculations for photoionization and dust radiative transfer in the galactic scale taking into account not only the global ISM structure but also small structures must be carried out in order to pin down the main origin of the extraplanar $\mathrm{H} \alpha$ emission. Wood et al. (2010) investigated models for the photoionization of the DIG in galaxies using hydrodynamic simulations of a supernova-driven ISM. However, the emission measure distributions in their simulations were found to be wider than those derived from $\mathrm{H} \alpha$ observations, implying the adopted ISM models are too porous to represent the realistic density structure of the ISM. The emission measure distribution or the $\mathrm{H} \alpha$ intensity distribution is directly coupled to the density distribution or porosity of the ISM, as noted in Seon (2009). Therefore, more extensive studies on photoionization models of the DIG are required to reproduce not only the high altitude $\mathrm{H} \alpha$ emission but also the observed distribution of emission measure.

Recently, Zhang et al. (2017) investigated the DIG using a sample of 365 face-on galaxies and concluded that ionization by evolved stars in the galactic halo with LI(N)ER-like emission is likely a major ionization source for DIG. An alternative scenario was proposed by Dong \& Draine (2011). In their model, the diffuse $\mathrm{H} \alpha$ emission is likely powered by runaway OB stars. We note that a time dependent photoionization model is needed to investigate the cooling and recombining gas model proposed by Dong \& Draine (2011), which is even more challenging than most of the photoionization models assuming a steady ionization state. Howk \& Savage (2000) suggested a scenario that may be relevant to the proposal of Dong \& Draine (2011). They found an anti-correlation between the FUV to $\mathrm{H} \alpha$ intensity ratio and the $\mathrm{H} \alpha$ intensity in face-on galaxies and proposed that photoionization of the diffuse ISM is maintained by late-type field OB stars. Seon et al. (2011) and Seon \& Witt (2012) also investigated this possibility. In the present study, it was found that the morphology at $\mathrm{H} \alpha$ is more compact and clumpier than that at FUV, implying an anti-correlation between the FUV to $\mathrm{H} \alpha$ intensity ratio and the $\mathrm{H} \alpha$ intensity.

The FUV halo emission could be either starlight from the stellar halo or a reflection nebula produced by scattering of FUV photons that escape the disk. The recent studies suggesting that evolved hot stars may contribute to the ionization of DIG (e.g., Zhang et al. 2017) might imply that some of the extraplanar FUV emission is also stellar in origin. One way to examine this possibility may be to compare the various correlations presented in this study with the stellar, vertical light distributions. Hodges-Kluck \& Bregman (2014) examined which scenario is more consistent with the data from the perspective of UV $-\mathrm{r}$ color in the halo, and SED fitting using dust models. They found that $\mathrm{UV}-\mathrm{r}$ colors and SEDs in the halos are more consistent with being a reflection nebula. Using dust radiative transfer models, Seon et al. (2014) and Shinn \& Seon (2015) could successfully explain the vertically extended FUV and NUV emissions as being due to dust-scattered starlight. The amount and scale height of eDust that were calculated from the radiative transfer models were found to be consistent with the observed vertical profile of FIR emission in NGC 891 (Bocchio et al. 2016). Polarization maps in the optical wavelengths can also trace large-scale galactic dust distributions. In edge-on galaxies, for instance NGC 891 and NGC 4565, extended optical polarization features were found in the halo regions above the galactic midplane (Scarrott et al. 1990: Scarrott \& Draper 1996 Fendt et al. 1996). If only a thin dust layer with a scale height of $\sim 0.2 \mathrm{kpc}$ is assumed, the polarization arising from scattering or dichroic extinction is predicted to be very low at high altitudes, and hence the extended polarization pattern cannot be explained (e.g., Bianchi et al. 1996: Wood \& Jones 1997, Peest et al. 2017). Therefore, the extended optical polarization indicates the existence of a thick dust disk. In a seperate paper (Seon 2018, submitted), we show that the extended optical polarization can be well explained by the extraplanar dust layer which was inferred from the observations of UV halos. Therefore, most of the extraplanar FUV emission measured in our galaxies can be attributed to scattered light rather than to direct starlight.

The simultaneous existence of the diffuse eDust and the extraplanar $\mathrm{H} \alpha$ emission suggests an interesting possibility that a large fraction of the $\mathrm{H} \alpha$ emission could originate from the galactic plane and is scattered by the eDust into sightlines of the galactic halo. Ferrara et al. (1996) and Barnes et al. (2015) investigated models for dust scattering of $\mathrm{H} \alpha$ photons by assuming only a thin dust disk, without taking into account eDust, and found that less than $\sim 20 \%$ of the total $\mathrm{H} \alpha$ intensity can be attributed to dust scattering. Here, it should be emphasized that the extraplanar FUV emission scattered by the eDust is more extended than the extraplanar $\mathrm{H} \alpha$ emission. The scattering cross-section at $\mathrm{H} \alpha$ is lower than that at FUV only by a factor of 1.9 for the Milky Way dust (Weingartner \& Draine 2001, Draine 2003). Therefore, there is no reason not to consider the possibility in which a substantial portion of the total extraplanar $\mathrm{H} \alpha$ emission is attributed to dust scattering by eDust. 
The most clear evidence on the existence of the ionized gas is provided by the pulsar dispersion measures. However, the pulsar dispersion measure alone provides only limited information (column density of electrons) on the ionized gas. The volume filling fraction and temperature of the DIG in the Milky Way were estimated from an "implicit" assumption that the pulsar dispersion measure and the $\mathrm{H} \alpha$ photons probe the same ionized medium (Reynolds 1989; Heiles 2001; Gaensler et al. 2008). Heiles (2001) argued that the DIG probed by the diffuse $\mathrm{H} \alpha$ emission is needed to be distinguished from the ionized gas that is traced by the pulsar dispersion measure. He showed that the pulsar dispersion measures are highly likely to be produced mainly by the warm ionized medium (WIM) predicted in the three phase model of McKee \& Ostriker (1977). In the thee phase model, the WIM is predicted to occupy relatively a small fraction of the ISM.

It has been argued that the scattering effect does not seem to be able to explain the observation that the ratios of forbidden lines to Balmer line such as $[\mathrm{N}$ II] $/ \mathrm{H} \alpha$ and [S II]/H $\alpha$ increase with the altitude of a galaxy (Reynolds 1985, 1987, Walterbos \& Braun 1994). However, Seon \& Witt (2012) suggested a potential solution to resolve this problem. The stellar continuum outside of bright H II regions is dominated by B- and A-type stars (Kennicutt 1992b a). Balmer absorption lines in the underlying stellar continuum and its scattered continuum background can give rise to underestimation of the $\mathrm{H} \alpha$ intensity and thus overestimation of the line ratios of forbidden lines. However, in recent spectroscopic studies of the DIG, the stellar continuum was fitted with stellar sythesis models before the emission line ratios were measured (Jones et al. 2017: Zhang et al. 2017). The resulting lines ratios in the DIG were found to be different from those in H II regions. Therefore, the present results do not imply that most of the $\mathrm{H} \alpha$ emission is caused by the scattered light. Instead, it is suggested that a larger fraction of the extraplanar $\mathrm{H} \alpha$ emission than that predicted by Ferrara et al. (1996) and Barnes et al. (2015) may be caused by scattered $\mathrm{H} \alpha$ photons. We, therefore, need to develop detailed models combining both photoionization and dust scattering to investigate the importance of the dust scattering by eDust. In a forthcoming paper, we will show how large fraction of the extraplanar $\mathrm{H} \alpha$ emission is attributable to the light scattered by the eDust and discuss the effect of dust-scattered $\mathrm{H} \alpha$ emission on the line ratios.

We now discuss the relationship between the FUV and $\mathrm{H} \alpha$ scale heights. If the total $\mathrm{H} \alpha$ intensity in the galactic halo originates from photoionized gas and the eDIG is uniformly mixed with the eDust, which is exponentially distributed with a scale height of $Z_{\mathrm{eDust}}$, then the scale height measured at $\mathrm{H} \alpha$ will be given by $\mathrm{Z}_{\mathrm{H} \alpha}=0.5 Z_{\mathrm{eDust}}$, which is denoted by blue triple-dot-dashed lines in Figures 2(c) and (d). This is because the emission measure is proportional to the square of electron density. Therefore, the relation between the two scale heights will provide a useful constraint in understanding the properties of the extraplanar ISM. In this paper, we found that $Z_{\mathrm{H} \alpha} \sim 0.74 Z_{\mathrm{FUV}}$, which appears to be inconsistent with that expected from photoionized gas. In the analyses of Seon et al. (2014) and Shinn \& Seon (2015), we found that the scale height of dust-scattered light $\left(Z_{\mathrm{FUV}}\right.$ or $\left.Z_{\mathrm{NUV}}\right)$ is similar to the intrinsic scale height of eDust
$\left(Z_{\text {eDust }}\right)$, but not always the same as the intrinsic value. The relation between the scale height of scattered light and the intrinsic scale height of eDust is not clear at this moment. Therefore, the relation $Z_{\mathrm{H} \alpha} \sim 0.74 Z_{\mathrm{FUV}}$ does not necessarily indicate that the $\mathrm{H} \alpha$ scale height is inconsistent with that expected from photoionized gas.

It is necessary to investigate radiative transfer models to better explain the present observations. The radiative transfer models could also provide the amount of dust expelled by the SF activities for our galaxies, as in Seon et al. (2014) and Shinn \& Seon (2015).

This research was supported by the Korea Astronomy and Space Science Institute under the R\&D program supervised by the Ministry of Science, ICT, and Future Planning of Korea. This research was also supported by the BK 21 plus program and Basic Science Research Program (2017R1D1A1B03031842) through the National Research Foundation (NRF) funded by the Ministry of Education of Korea. K.-I. Seon was supported by the National Research Foundation of Korea (NRF) grant funded by the Korea government (MSIP) (No. 2017R1A2B4008291). K.-I. Seon thanks Hyunjin Jung and Changhee Rhee for helpful discussion on the star-formation rates and spectral energy distribution of spiral galaxies.

\section{REFERENCES}

Alton, P. B., Rand, R. J., Xilouris, E. M., et al. 2000a, A\&AS, 145,83

Alton, P. B., Xilouris, E. M., Bianchi, S., Davies, J., \& Kylafis, N. 2000b, A\&A, 356, 795

Alton, P. B., Xilouris, E. M., Misiriotis, A., Dasyra, K. M., \& Dumke, M. 2004, A\&A, 425, 109

Baes, M., \& Viaene, S. 2016, A\&A, 587, A86

Barnes, J. E., Wood, K., Hill, A. S., \& Haffner, L. M. 2015, MNRAS, 447, 559

Bertin, E., \& Arnouts, S. 1996, A\&AS, 117, 393

Bianchi, S., Ferrara, A., \& Giovanardi, C. 1996, ApJ, 465, 127

Bocchio, M., Bianchi, S., Hunt, L. K., \& Schneider, R. 2016, A\&A, 586, A8

Burgdorf, M., Ashby, M. L. N., \& Williams, R. 2007, ApJ, 668, 918

Cecil, G., Bland-Hawthorn, J., \& Veilleux, S. 2002, ApJ, 576, 745

Dale, D. A., Cohen, S. A., Johnson, L. C., et al. 2009, ApJ, 703, 517

De Geyter, G., Baes, M., Camps, P., et al. 2014, MNRAS, 441, 869

Dettmar, R.-J. 2005, Extra-Planar Gas, 331, 155

Domgorgen, H., \& Mathis, J. S. 1994, ApJ, 428, 647

Dong, R., \& Draine, B. T. 2011, ApJ, 727, 35

Draine, B. T. 2003, ApJ, 598, 1017

Fendt, C., Beck, R., Lesch, H., \& Neininger, N. 1996, A\&A, 308, 713

Ferguson, A. M. N., Wyse, R. F. G., Gallagher, J. S. I., \& Hunter, D. A. 1996, AJ, 111, 2265

Ferrara, A., Bianchi, S., Dettmar, R.-J., \& Giovanardi, C. 1996, ApJL, 467, L69

Ferrara, A., Ferrini, F., Barsella, B., \& Franco, J. 1991, ApJ, 381, 137

Flores-Fajardo, N., Morisset, C., Stasinska, G., \& Binette, L. 2011, MNRAS, 415, 2182

Franco, J., Ferrini, F., Barsella, B., \& Ferrara, A. 1991, ApJ, 366, 443

Gaensler, B. M., Madsen, G. J., Chatterjee, S., \& Mao, S. A 2008, PASA, 25, 184

Gavazzi, G., Boselli, A., Donati, A., Franzetti, P., \& Scodeggio, M. 2003, A\&A, 400, 451

Gavazzi, G., Fumagalli, M., Galardo, V., et al. 2012, A\&A, 545, A16 
Greenberg, J. M., Ferrini, F., Barsella, B., \& Aiello, S. 1987, Nature, 327, 214

Haffner, L. M., Dettmar, R.-J., Beckman, J. E., et al. 2009, RMP, 81,969

Heiles, C. 2001, Tetons 4: Galactic Structure, 231, 294

Ho, I. T., Medling, A. M., Bland-Hawthorn, J., et al. 2016, MNRAS, 457, 1257

Hodges-Kluck, E., \& Bregman, J. N. 2014, ApJ, 789, 131

Hodges-Kluck, E., Cafmeyer, J., \& Bregman, J. N. 2016, ApJ, 833,58

Hoopes, C. G., \& Walterbos, R. A. M. 2000, ApJ, 541, 597

Hoopes, C. G., Walterbos, R. A. M., \& Bothun, G. D. 2001, ApJ, 559,878

Hoopes, C. G., Heckman, T. M., Strickland, D. K., et al. 2005, ApJ, 619, L99

Howk, J. C., \& Savage, B. D. 1997, AJ, 114, 2463

- 1999, AJ, 117, 2077

-. 2000, AJ, 119, 644

Irwin, J. A., \& Madden, S. C. 2006, A\&A, 445, 123

James, P. A., Shane, N. S., Beckman, J. E., et al. 2004, A\&A, 414, 23

Jones, A., Kauffmann, G., D’Souza, R., et al. 2017, A\&A, 599, A141

Kaneda, H., Yamagishi, M., Suzuki, T., \& Onaka, T. 2009, ApJ, 698, L125

Kennicutt, R. C. J. 1992a, ApJS, 79, 255

-. 1992b, ApJ, 388, 310

-. 1998, ApJ, 498, 541

Kennicutt, R. C. J., Lee, J. C., Funes, S. J. J. G., Sakai, S., \& Akiyama, S. 2008, ApJS, 178, 247

Kennicutt, R. C. J., Armus, L., Bendo, G., et al. 2003, PASP, 115,928

Mac Low, M.-M., \& Ferrara, A. 1999, ApJ, 513, 142

Martin, D. C., Fanson, J., Schiminovich, D., et al. 2005, ApJ, 619, L1

McCormick, A., Veilleux, S., \& Rupke, D. S. N. 2013, ApJ, 774, 126

McKee, C. F., \& Ostriker, J. P. 1977, ApJ, 218, 148

Meurer, G. R., Hanish, D. J., Ferguson, H. C., et al. 2006, ApJS, 165,307

Miller, S. T., \& Veilleux, S. 2003a, ApJS, 148, 383

-. 2003b, ApJ, 592, 79

Morrissey, P., Schiminovich, D., Barlow, T. A., et al. 2005, ApJ, 619, L7

Morrissey, P., Conrow, T., Barlow, T. A., et al. 2007, ApJS, 173, 682

Moshir, M., \& al, e. 1990, IRAS Faint Source Catalogue

Moustakas, J., Kennicutt, R. C. J., Tremonti, C. A., et al. 2010, ApJS, 190, 233

Peest, C., Camps, P., Stalevski, M., Baes, M., \& Siebenmorgen, R. 2017, A\&A, 601, A92

Putman, M. E., Peek, J. E. G., \& Joung, M. R. 2012, ARA\&A, 50,491
Rand, R. J. 1996, ApJ, 462, 712

Reynolds, R. J. 1984, ApJ, 282, 191

-. 1985, ApJ, 294, 256

-. 1987, ApJ, 323, 118

-. 1989, ApJ, 339, L29

Rice, W., Lonsdale, C. J., Soifer, B. T., et al. 1988, ApJS, 68, 91

Rossa, J., \& Dettmar, R.-J. 2000, A\&A, 359, 433

-. 2003a, A\&A, 406, 493

- . 2003b, A\&A, 406, 505

Rossa, J., Dettmar, R.-J., Walterbos, R. A. M., \& Norman, C. A. 2004, AJ, 128, 674

Rueff, K. M., Howk, J. C., Pitterle, M., et al. 2013, AJ, 145, 62

Sandin, C. 2014, A\&A, 567, A97

-. 2015, A\&A, 577, A106

Scarrott, S. M., \& Draper, P. W. 1996, MNRAS, 278, 519

Scarrott, S. M., Rolph, C. D., \& Semple, D. P. 1990, in IN: Galactic and intergalactic magnetic fields; Proceedings of the 140th Symposium of IAU, Durham, University, England, $245-251$

Seon, K.-I. 2009, ApJ, 703, 1159

Seon, K.-I., Witt, A., Kim, I.-J., et al. 2011, ApJ, 743, 188

Seon, K.-I., \& Witt, A. N. 2012, ApJ, 758, 109

Seon, K.-I., Witt, A. N., Shinn, J.-H., \& Kim, I.-J. 2014, ApJ, $785, \mathrm{~L} 18$

Shinn, J.-H., \& Seon, K.-I. 2015, ApJ, 815, 133

Strickland, D. K., Heckman, T. M., Colbert, E. J. M., Hoopes, C. G., \& Weaver, K. A. 2004a, ApJS, 151, 193

-. 2004b, ApJ, 606, 829

Thompson, T. W. J., Howk, J. C., \& Savage, B. D. 2004, AJ, 128, 662

Veilleux, S., Cecil, G., \& Bland-Hawthorn, J. 2005, ARA\&A, 43, 769

Verstappen, J., Fritz, J., Baes, M., et al. 2013, A\&A, 556, 54

Walterbos, R. A. M., \& Braun, R. 1994, ApJ, 431, 156

Weingartner, J. C., \& Draine, B. T. 2001, ApJ, 548, 296

Wood, K., Hill, A. S., Joung, M. R., et al. 2010, ApJ, 721, 1397

Wood, K., \& Jones, T. J. 1997, AJ, 114, 1405

Wood, K., \& Mathis, J. S. 2004, MNRAS, 353, 1126

Wood, K., \& Reynolds, R. J. 1999, ApJ, 525, 799

Xilouris, E. M., Alton, P. B., Davies, J. I., et al. 1998, A\&A, 331, 894

Xilouris, E. M., Byun, Y. I., Kylafis, N. D., Paleologou, E. V., \& Papamastorakis, J. 1999, A\&A, 344, 868

Xilouris, E. M., Kylafis, N. D., Papamastorakis, J., Paleologou, E. V., \& Haerendel, G. 1997, A\&A, 325, 135

Zhang, K., Yan, R., Bundy, K., et al. 2017, MNRAS, 466, 3217

Zurita, A., Beckman, J. E., Rozas, M., \& Ryder, S. 2002, A\&A, 386,801 
Table 1

Galaxy samples.

\begin{tabular}{|c|c|c|c|c|c|c|c|c|c|c|c|}
\hline No. & Name & Morphology & $\begin{array}{l}\text { Gal.Lon. } \\
\text { (degree) }\end{array}$ & $\begin{array}{l}\text { Gal.Lat. } \\
\text { (degree) }\end{array}$ & $\begin{array}{l}\text { Distance } \\
(\mathrm{Mpc})\end{array}$ & $\begin{array}{c}\mathrm{D}_{\text {major }} \\
(\text { arcmin })\end{array}$ & $\begin{array}{l}\mathrm{D}_{25} \\
(\mathrm{kpc})\end{array}$ & $\begin{array}{c}\mathrm{L}_{\mathrm{H} \alpha} \\
\left(10^{40} \text { erg s s}^{-1}\right)\end{array}$ & $\begin{array}{c}\mathrm{SFR}_{\mathrm{H} \alpha} \\
\left(\mathrm{M}_{\odot} \mathrm{yr}^{-1}\right)\end{array}$ & $\begin{array}{c}\mathrm{SFR}_{\mathrm{FIR}} \\
\left(\mathrm{M}_{\odot} \mathrm{yr}^{-1}\right)\end{array}$ & ref \\
\hline 1 & UGCA 442 & $\mathrm{SB}(\mathrm{s}) \mathrm{m} ?$ & 10.70 & -74.53 & 5.55 & 6.38 & 10.3 & 0.147 & 0.0117 & 0.0009 & SINGG \\
\hline 2 & NGC 5951 & SBc? & 23.52 & 50.45 & 26.58 & 3.50 & 27.1 & 3.616 & 0.2870 & 0.1652 & $\mathrm{H} \alpha 3$ \\
\hline 3 & NGC 5107 & $\mathrm{SB}(\mathrm{s}) \mathrm{d} ?$ & 96.01 & 76.98 & 18.54 & 1.70 & 9.2 & & & 0.0892 & $\mathrm{H} \alpha \mathrm{GS}$ \\
\hline 4 & NGC 5229 & $\mathrm{SB}(\mathrm{s}) \mathrm{d}$ ? & 103.95 & 67.61 & 9.32 & 3.58 & 9.7 & 0.308 & 0.0244 & 0.0076 & LVL \\
\hline 5 & UGC 08313 & $\mathrm{SB}(\mathrm{s}) \mathrm{c} ?$ & 107.46 & 74.24 & 9.32 & 1.91 & 5.2 & 0.377 & 0.0299 & 0.0050 & LVL \\
\hline 6 & NGC 5023 & Scd? & 110.38 & 72.58 & 9.36 & 7.28 & 19.8 & 0.798 & 0.0633 & 0.0204 & LVL \\
\hline 7 & NGC 0493 & $\mathrm{SAB}(\mathrm{s}) \mathrm{cd} ?$ & 138.91 & -60.97 & 21.94 & 3.40 & 21.7 & & & 0.2275 & $\mathrm{H} \alpha \mathrm{GS}$ \\
\hline 8 & NGC 0891 & $\mathrm{SA}(\mathrm{s}) \mathrm{b} ?$ & 140.38 & -17.41 & 9.59 & 13.50 & 37.7 & 5.866 & 0.4655 & 1.3909 & Rossa \\
\hline 9 & NGC 0784 & SBdm? & 140.90 & -31.59 & 4.21 & 6.60 & 8.1 & 0.408 & 0.0323 & 0.0034 & LVL \\
\hline 10 & NGC 4631 & $\mathrm{SB}(\mathrm{s}) \mathrm{d}$ & 142.81 & 84.22 & 5.16 & 15.50 & 23.3 & 11.414 & 0.9059 & 0.4946 & SINGS \\
\hline 11 & NGC 4144 & $\mathrm{SAB}(\mathrm{s}) \mathrm{cd} ?$ & 143.17 & 69.01 & 6.14 & 6.00 & 10.7 & 0.962 & 0.0764 & 0.0193 & LVL \\
\hline 12 & NGC 0803 & $\mathrm{SA}(\mathrm{s}) \mathrm{c} ?$ & 147.17 & -43.41 & 22.29 & 3.00 & 19.5 & & & 0.1573 & $\mathrm{H} \alpha \mathrm{GS}$ \\
\hline 13 & NGC 4244 & $\mathrm{SA}(\mathrm{s}) \mathrm{cd}$ ? & 154.57 & 77.16 & 4.11 & 19.38 & 23.2 & 1.023 & 0.0812 & 0.0308 & LVL \\
\hline 14 & IC 2233 & $\mathrm{SB}(\mathrm{s}) \mathrm{d} ?$ & 174.12 & 33.06 & 12.27 & 5.17 & 18.5 & 1.723 & 0.1368 & 0.0187 & LVL \\
\hline 15 & NGC 3432 & $\mathrm{SB}(\mathrm{s}) \mathrm{m}$ & 184.77 & 63.16 & 10.98 & 6.80 & 21.7 & 6.732 & 0.5343 & 0.2260 & LVL \\
\hline 16 & NGC 4020 & SBd? & 193.90 & 78.05 & 12.04 & 2.24 & 7.8 & 1.124 & 0.0892 & 0.0444 & LVL \\
\hline 17 & NGC 3510 & $\mathrm{SB}(\mathrm{s}) \mathrm{m}$ & 202.36 & 66.21 & 13.95 & 4.35 & 17.7 & 2.380 & 0.1889 & 0.0401 & LVL \\
\hline 18 & NGC 3190 & $\mathrm{SA}(\mathrm{s}) \mathrm{a}$ pec & 213.04 & 54.85 & 24.35 & 4.40 & 31.2 & & & 0.7462 & SINGS \\
\hline 19 & NGC 3628 & Sb pec & 240.85 & 64.78 & 9.85 & 14.80 & 42.4 & 4.130 & 0.3278 & 1.5407 & $\mathrm{H} \alpha 3$ \\
\hline 20 & UGCA 193 & Sd? & 245.64 & 37.43 & 11.00 & 4.31 & 13.8 & 0.270 & 0.0215 & 0.0012 & SINGG \\
\hline 21 & NGC 3365 & Scd? & 247.75 & 50.76 & 17.53 & 4.84 & 24.7 & 2.122 & 0.1684 & 0.0849 & SINGG \\
\hline 22 & NGC 4455 & $\mathrm{SB}(\mathrm{s}) \mathrm{d} ?$ & 251.64 & 83.29 & 9.13 & 2.80 & 7.4 & 0.888 & 0.0705 & 0.0138 & LVL \\
\hline 23 & ESO 249- G 035 & SBcd? & 252.61 & -48.67 & 22.49 & 1.31 & 8.6 & 0.175 & 0.0139 & 0.0018 & SINGG \\
\hline 24 & IC 2000 & $\mathrm{SB}(\mathrm{s}) \mathrm{cd} ?$ & 257.65 & -49.60 & 19.39 & 4.10 & 23.1 & 2.980 & 0.2365 & 0.1064 & SINGG \\
\hline 25 & IC 1959 & $\mathrm{SB}(\mathrm{s}) \mathrm{m} ?$ & 261.28 & -51.54 & 7.90 & 2.80 & 6.4 & 0.667 & 0.0530 & 0.0108 & SINGG \\
\hline 26 & NGC 1311 & $\mathrm{SB}(\mathrm{s}) \mathrm{m} ?$ & 265.29 & -52.66 & 4.96 & 3.00 & 4.3 & 0.214 & 0.0170 & 0.0032 & SINGG \\
\hline 27 & NGC 4313 & SA(rs)ab? & 277.74 & 73.25 & 14.62 & 4.99 & 21.2 & 0.811 & 0.0644 & 0.1065 & $\mathrm{H} \alpha 3$ \\
\hline 28 & NGC 4388 & $\mathrm{SA}(\mathrm{s}) \mathrm{b} ?$ & 279.12 & 74.34 & 19.50 & 4.84 & 27.5 & & & 1.1619 & Rossa \\
\hline 29 & NGC 4469 & SB0/a?(s) & 286.13 & 70.90 & 16.75 & 2.50 & 12.2 & & & 0.1130 & $\mathrm{H} \alpha \mathrm{GS}$ \\
\hline 30 & NGC 4866 & $\mathrm{SA}^{\wedge}+(\mathrm{r}) ?$ & 311.54 & 76.91 & 23.09 & 6.30 & 42.3 & 2.792 & 0.2216 & 0.0515 & $\mathrm{H} \alpha 3$ \\
\hline 31 & IC 5176 & SAB $(\mathrm{s}) \mathrm{bc} ?$ & 323.00 & -43.69 & 26.86 & 6.05 & 47.3 & 4.240 & 0.3365 & 0.9423 & SINGG \\
\hline 32 & IC 5052 & SBd? & 325.18 & -35.81 & 7.46 & 5.90 & 12.8 & 2.263 & 0.1796 & 0.0395 & SINGG \\
\hline 33 & IC 4951 & $\mathrm{SB}(\mathrm{s}) \mathrm{dm} ?$ & 334.89 & -32.85 & 8.97 & 2.80 & 7.3 & 0.278 & 0.0221 & 0.0046 & SINGG \\
\hline 34 & NGC 5348 & SBbc? & 340.03 & 63.49 & 18.44 & 3.50 & 18.8 & 1.481 & 0.1176 & 0.0357 & $\mathrm{H} \alpha 3$ \\
\hline 35 & NGC 5356 & SABbc? & 340.53 & 63.47 & 23.89 & 3.71 & 25.8 & 1.930 & 0.1532 & 0.1485 & $\mathrm{H} \alpha 3$ \\
\hline 36 & NGC 7090 & SBc? & 341.30 & -45.39 & 7.76 & 7.40 & 16.7 & 2.831 & 0.2247 & 0.1370 & LVL \\
\hline 37 & NGC $7412 \mathrm{~A}$ & SBdm? & 351.39 & -62.04 & 9.74 & 5.11 & 14.5 & 0.212 & 0.0168 & 0.0025 & SINGG \\
\hline 38 & ESO 347- G 017 & $\mathrm{SB}(\mathrm{s}) \mathrm{m} ?$ & 357.78 & -69.49 & 7.89 & 1.60 & 3.7 & 0.175 & 0.0139 & 0.0042 & SINGG \\
\hline
\end{tabular}

Column (1): the running index number. Column (2): galaxy name taken from the NED's preferred object name. Column (3): galaxy morphology. Column (4): Galactic longitude. Column (5): Galactic latitude. Column (6): average value of the redshift-independent distances. Column (7): major axis diameter in arcmin. Column (8): major axis diameter in kpc calculated using Column (6) and (7): Column (9): integrated $\mathrm{H} \alpha$ luminosity taken from the reference papers. Column (10): star formation rate based on the H $\alpha$ luminosity. Column (11): star formation rate estimated using the FIR luminosity. 
Table 2

Scale heights of the sample galaxies.

\begin{tabular}{|c|c|c|c|c|c|c|c|c|c|}
\hline \multirow{2}{*}{ No. } & \multirow{2}{*}{ Name } & \multirow{2}{*}{ Group } & \multirow{2}{*}{$\begin{array}{c}\mathrm{SFR}_{\mathrm{FIR}} \\
\left(\mathrm{M}_{\odot} \mathrm{yr}^{-1}\right)\end{array}$} & \multicolumn{6}{|c|}{ scale height (kpc) } \\
\hline & & & & $\mathrm{H} \alpha$ & $\mathrm{H} \alpha$ err & FUV & FUV err & NUV & NUV err \\
\hline 1 & UGCA 442 & A & 0.0009 & 0.138 & 0.005 & 0.216 & 0.007 & 0.318 & 0.029 \\
\hline 2 & NGC 5951 & $\mathrm{~B}$ & 0.1652 & 0.595 & 0.043 & 0.818 & 0.134 & 0.770 & 0.141 \\
\hline 3 & NGC 5107 & B & 0.0892 & 0.319 & 0.018 & 0.442 & 0.013 & 0.538 & 0.029 \\
\hline 4 & NGC 5229 & A & 0.0076 & 0.136 & 0.293 & 0.198 & 0.018 & 0.293 & 0.048 \\
\hline 5 & UGC 08313 & A & 0.0050 & 0.194 & 0.017 & 0.160 & 0.006 & 0.201 & 0.014 \\
\hline 6 & NGC 5023 & A & 0.0204 & 0.238 & 0.012 & 0.302 & 0.012 & 0.340 & 0.027 \\
\hline 7 & NGC 0493 & B & 0.2275 & 1.068 & 0.077 & 0.811 & 0.050 & 0.801 & 0.095 \\
\hline 8 & NGC 0891 & B & 1.3909 & 0.821 & 0.052 & 1.778 & 0.462 & 1.340 & 0.497 \\
\hline 9 & NGC 0784 & A & 0.0034 & 0.180 & 0.016 & 0.171 & 0.005 & 0.203 & 0.008 \\
\hline 10 & NGC 4631 & B & 0.4946 & 0.266 & 0.045 & 0.979 & 0.018 & 0.655 & 0.025 \\
\hline 11 & NGC 4144 & A & 0.0193 & 0.197 & 0.011 & 0.272 & 0.010 & 0.308 & 0.024 \\
\hline 12 & NGC 0803 & $\mathrm{~B}$ & 0.1573 & 0.890 & 0.121 & 1.178 & 0.047 & 1.229 & 0.117 \\
\hline 13 & NGC 4244 & A & 0.0308 & 0.288 & 0.040 & 0.302 & 0.008 & 0.254 & 0.012 \\
\hline 14 & IC 2233 & A & 0.0187 & 0.229 & 0.008 & 0.245 & 0.005 & 0.271 & 0.010 \\
\hline 15 & NGC 3432 & B & 0.2260 & 0.308 & 0.003 & 0.697 & 0.013 & 0.562 & 0.020 \\
\hline 16 & NGC 4020 & B & 0.0444 & 0.293 & 0.010 & 0.578 & 0.049 & 0.672 & 0.131 \\
\hline 17 & NGC 3510 & B & 0.0401 & 0.322 & 0.014 & 0.478 & 0.011 & 0.462 & 0.022 \\
\hline 18 & NGC 3190 & B & 0.7462 & 0.737 & 0.293 & 2.035 & 0.239 & 1.973 & 0.263 \\
\hline 19 & NGC 3628 & B & 1.5407 & 0.862 & 0.015 & 1.529 & 0.064 & 1.654 & 0.122 \\
\hline 20 & UGCA 193 & A & 0.0012 & 0.222 & 0.018 & 0.162 & 0.020 & 0.211 & 0.043 \\
\hline 21 & NGC 3365 & B & 0.0849 & 0.533 & 0.028 & 0.500 & 0.055 & 0.509 & 0.086 \\
\hline 22 & NGC 4455 & A & 0.0138 & 0.218 & 0.008 & 0.303 & 0.013 & 0.331 & 0.025 \\
\hline 23 & ESO 249- G 035 & A & 0.0018 & 0.225 & 0.039 & 0.220 & 0.014 & 0.308 & 0.032 \\
\hline 24 & IC 2000 & B & 0.1064 & 0.634 & 0.054 & 0.551 & 0.055 & 0.679 & 0.095 \\
\hline 25 & IC 1959 & A & 0.0108 & 0.203 & 0.009 & 0.212 & 0.007 & 0.249 & 0.018 \\
\hline 26 & NGC 1311 & A & 0.0032 & 0.166 & 0.002 & 0.136 & 0.004 & 0.167 & 0.007 \\
\hline 27 & NGC 4313 & B & 0.1065 & 0.431 & 0.027 & 0.473 & 0.073 & 0.808 & 0.117 \\
\hline 28 & NGC 4388 & B & 1.1619 & 1.361 & 0.052 & 1.307 & 0.072 & 1.633 & 0.126 \\
\hline 29 & NGC 4469 & B & 0.1130 & 0.944 & 0.155 & 0.734 & 0.109 & 0.881 & 0.152 \\
\hline 30 & NGC 4866 & B & 0.0515 & 0.677 & 0.028 & 1.260 & 0.137 & 1.490 & 0.313 \\
\hline 31 & IC 5176 & B & 0.9423 & 0.618 & 0.008 & 0.802 & 0.052 & 0.794 & 0.096 \\
\hline 32 & IC 5052 & A & 0.0395 & 0.347 & 0.008 & 0.333 & 0.016 & 0.360 & 0.022 \\
\hline 33 & IC 4951 & A & 0.0046 & 0.188 & 0.005 & 0.211 & 0.008 & 0.241 & 0.014 \\
\hline 34 & NGC 5348 & B & 0.0357 & 0.245 & 0.044 & 0.402 & 0.034 & 0.473 & 0.084 \\
\hline 35 & NGC 5356 & B & 0.1485 & 0.307 & 0.027 & 0.915 & 0.177 & 1.341 & 0.449 \\
\hline 36 & NGC 7090 & B & 0.1370 & 0.627 & 0.006 & 0.911 & 0.097 & 0.727 & 0.114 \\
\hline 37 & NGC $7412 \mathrm{~A}$ & A & 0.0025 & 0.169 & 0.010 & 0.216 & 0.007 & 0.273 & 0.030 \\
\hline 38 & ESO 347- G 017 & A & 0.0042 & 0.175 & 0.007 & 0.170 & 0.007 & 0.230 & 0.020 \\
\hline
\end{tabular}



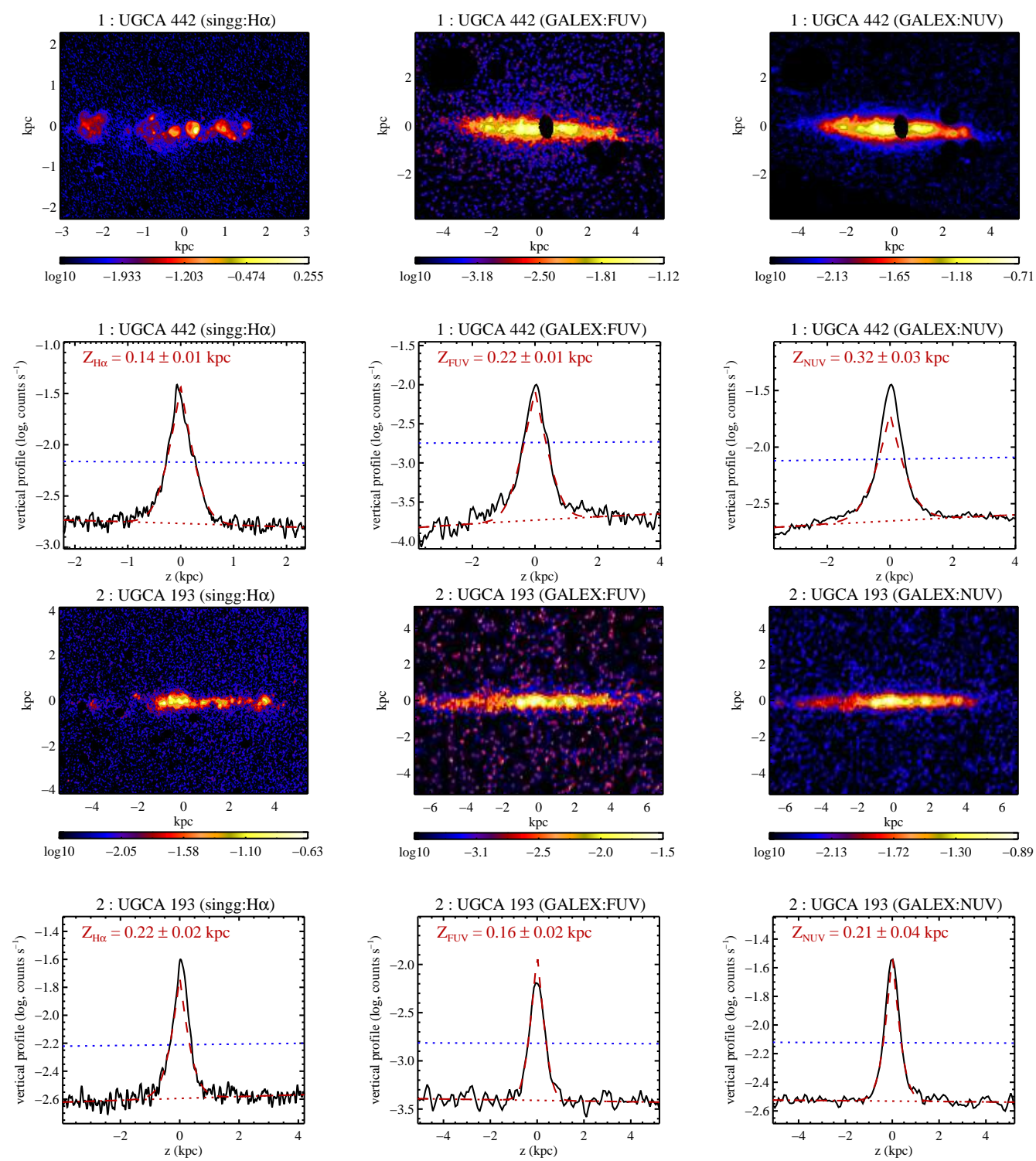

Figure 1. (left top) H $\alpha$ emission map, (middle top) GALEX FUV map, (right top) GALEX NUV map, (left bottom) H $\alpha$ vertical profile, (middle bottom) FUV vertical profile, and (right bottom) NUV vertical profile for each galaxy. In the vertical profiles, the red dotted lines indicate the background levels. The red dashed lines denote the best fit exponential function for the vertical profiles. The horizontal blue dotted lines are the line to distinguish the bright galactic plane region from the diffuse extraplanar region of the galaxies. 

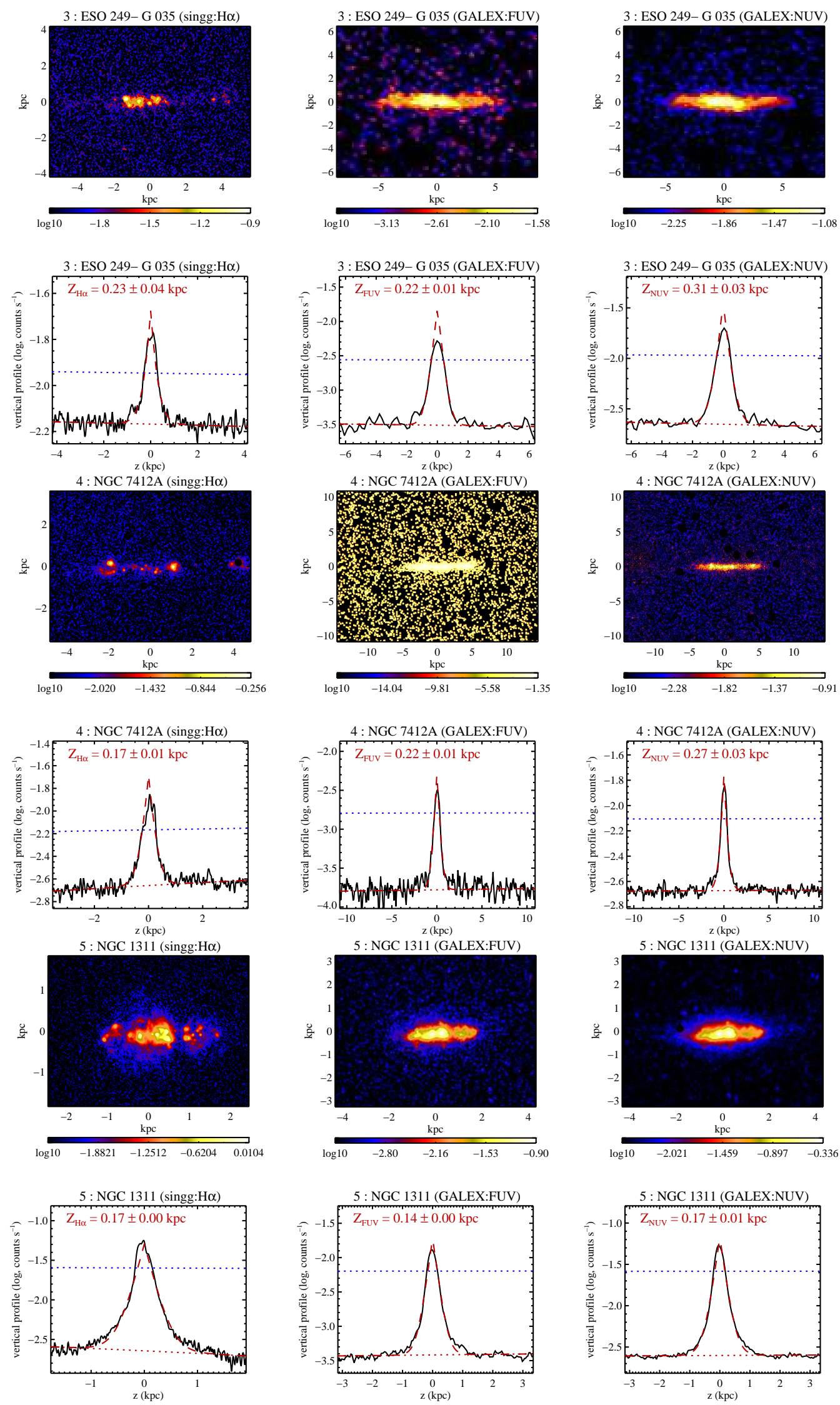

Figure 1. Continued. 

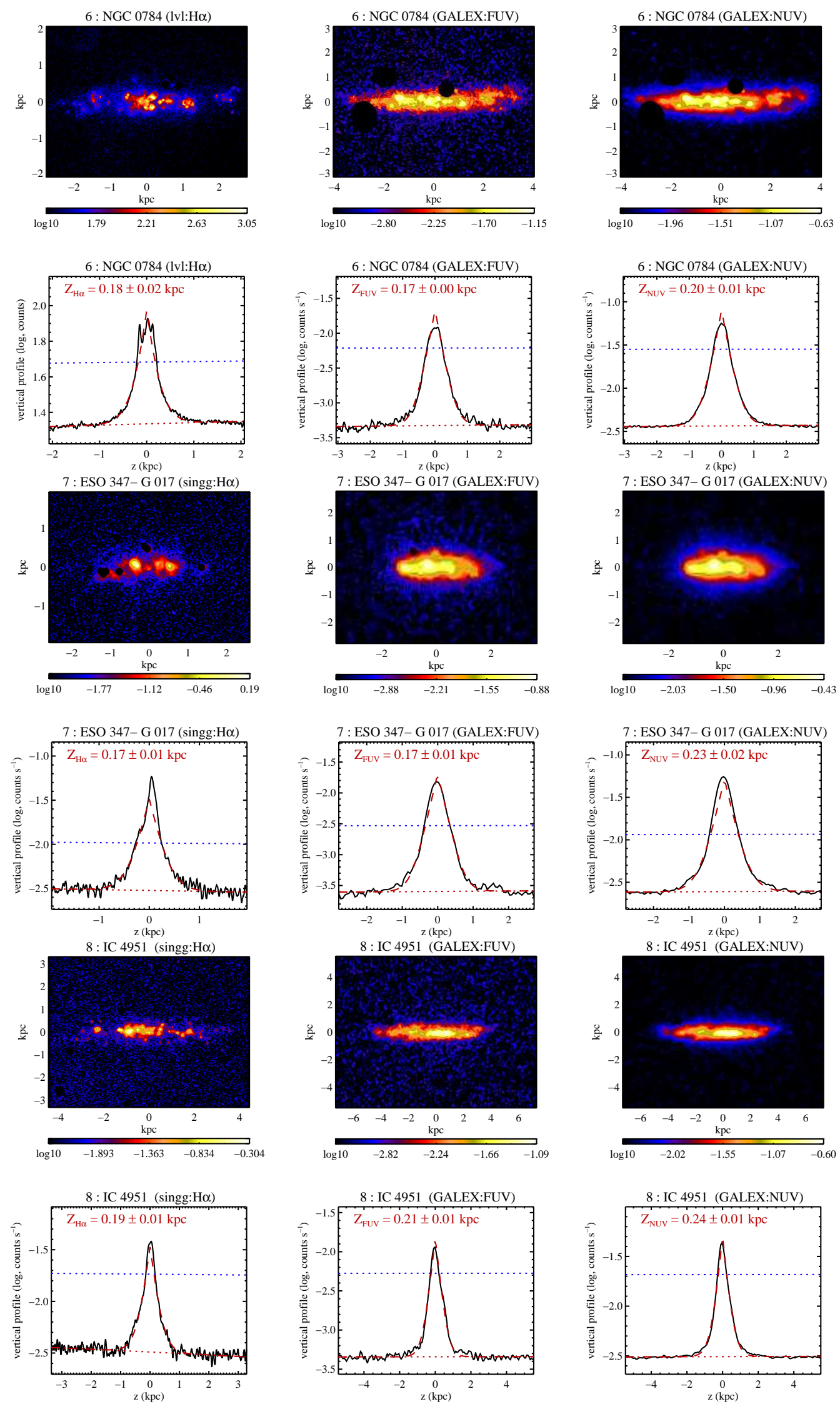

Figure 1. Continued. 

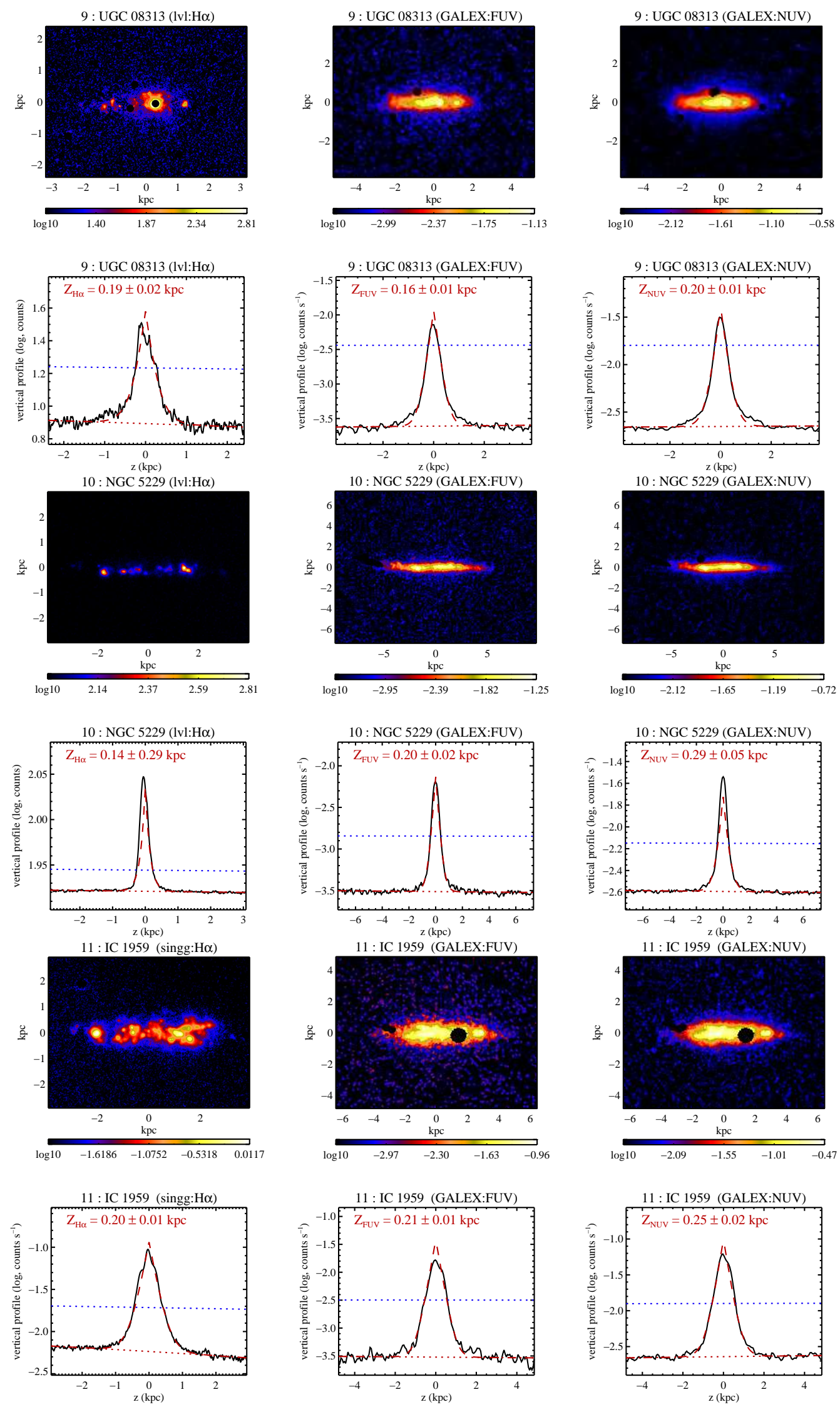

Figure 1. Continued. 

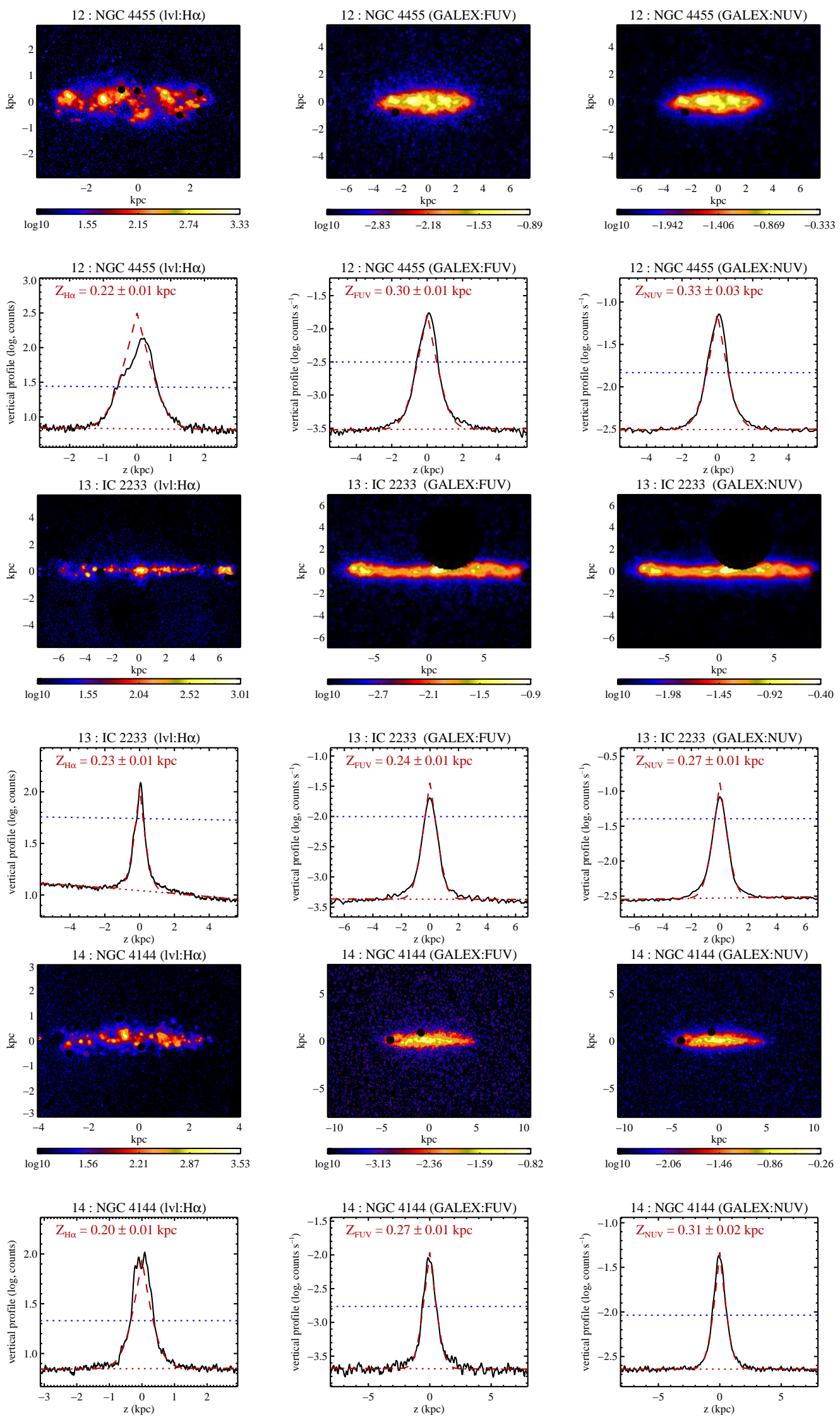

Figure 1. Continued. 

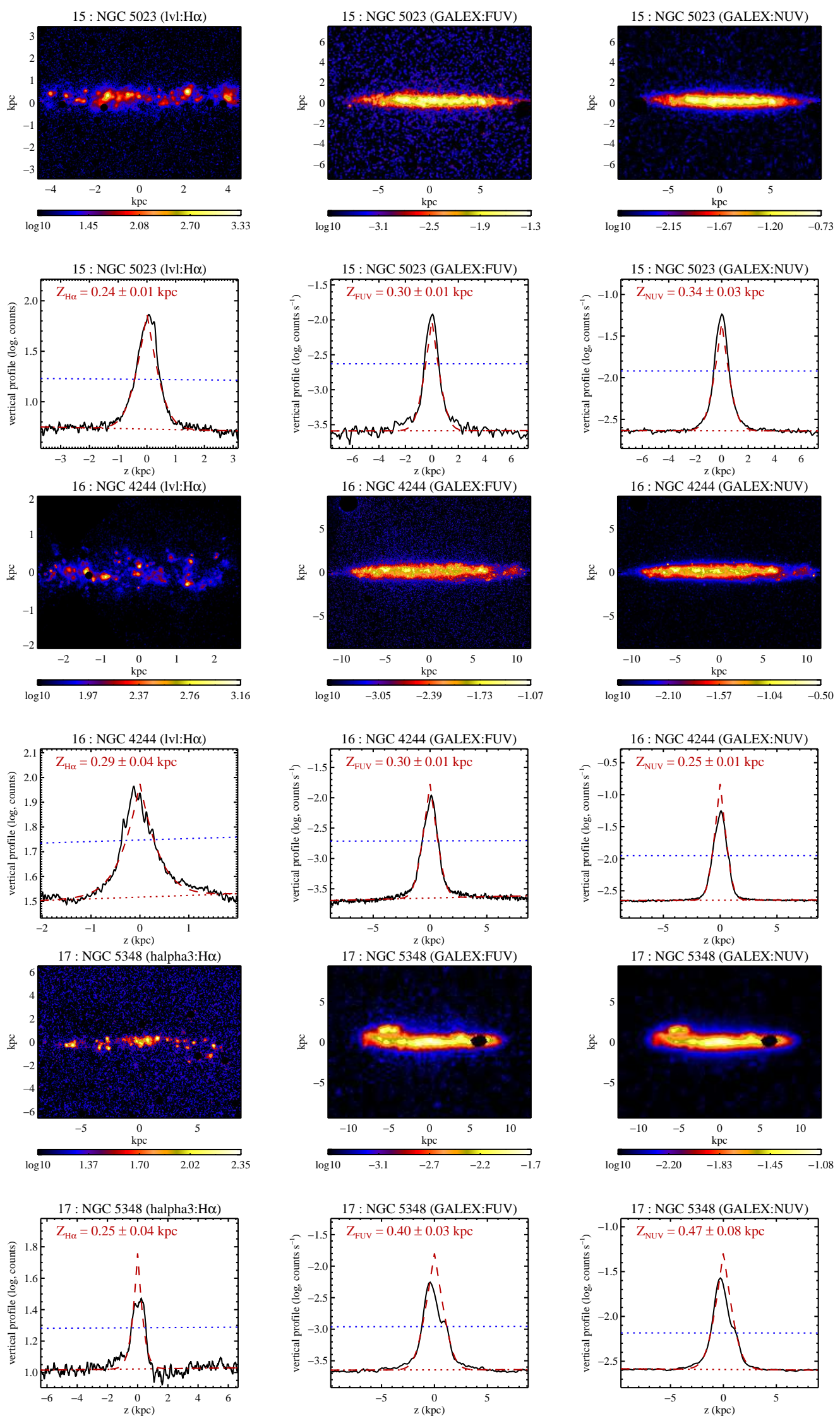

Figure 1. Continued. 

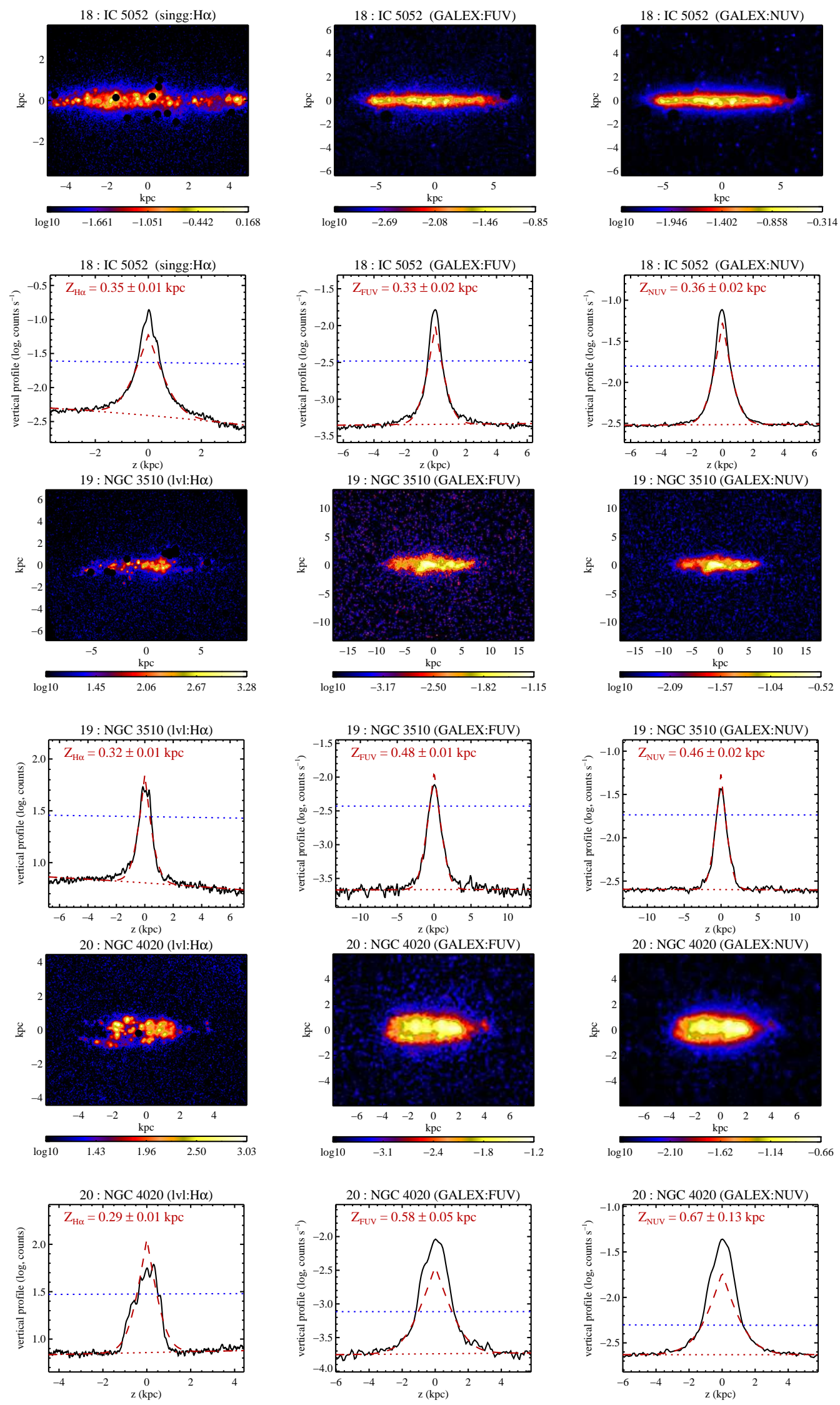

Figure 1. Continued. 

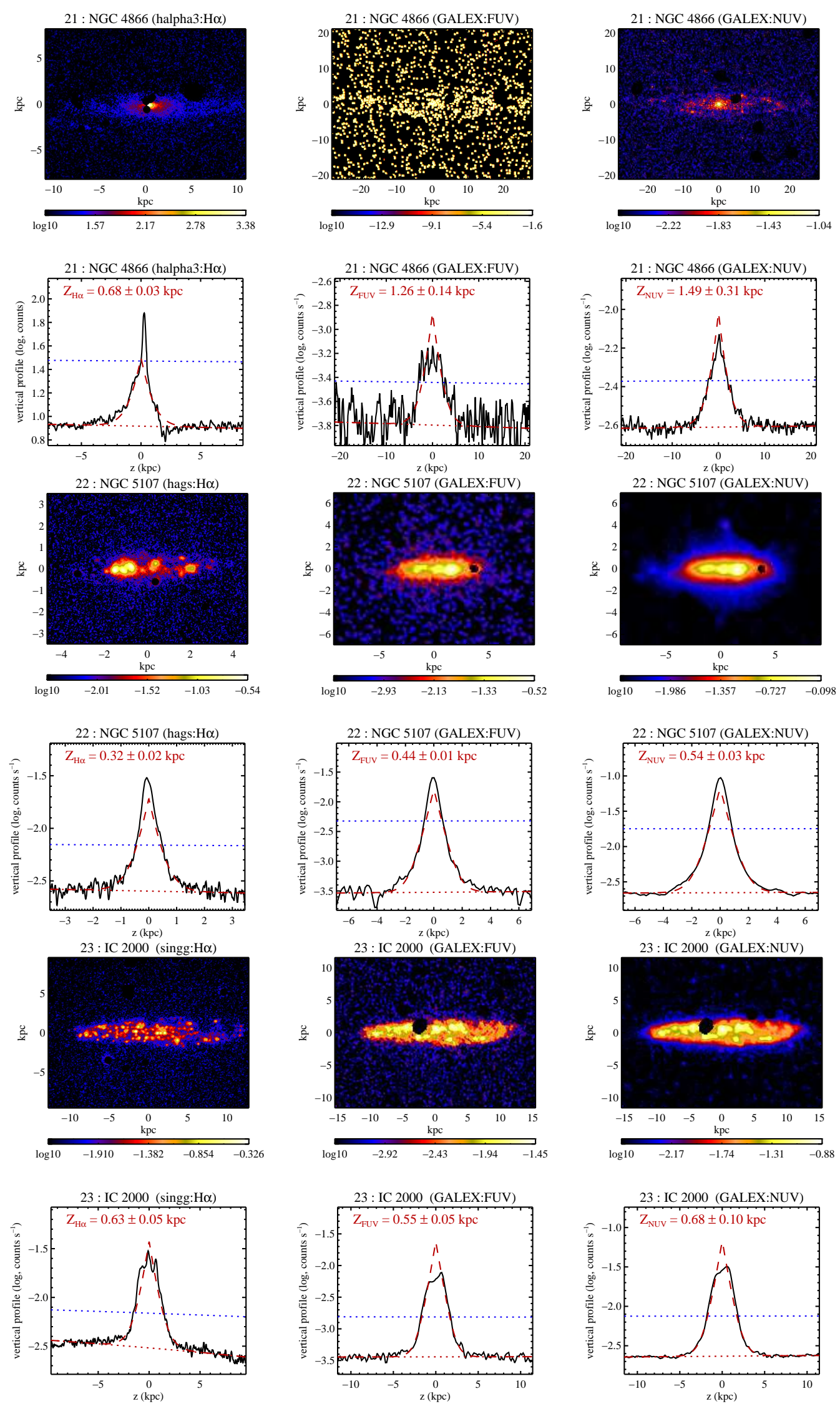

Figure 1. Continued. 

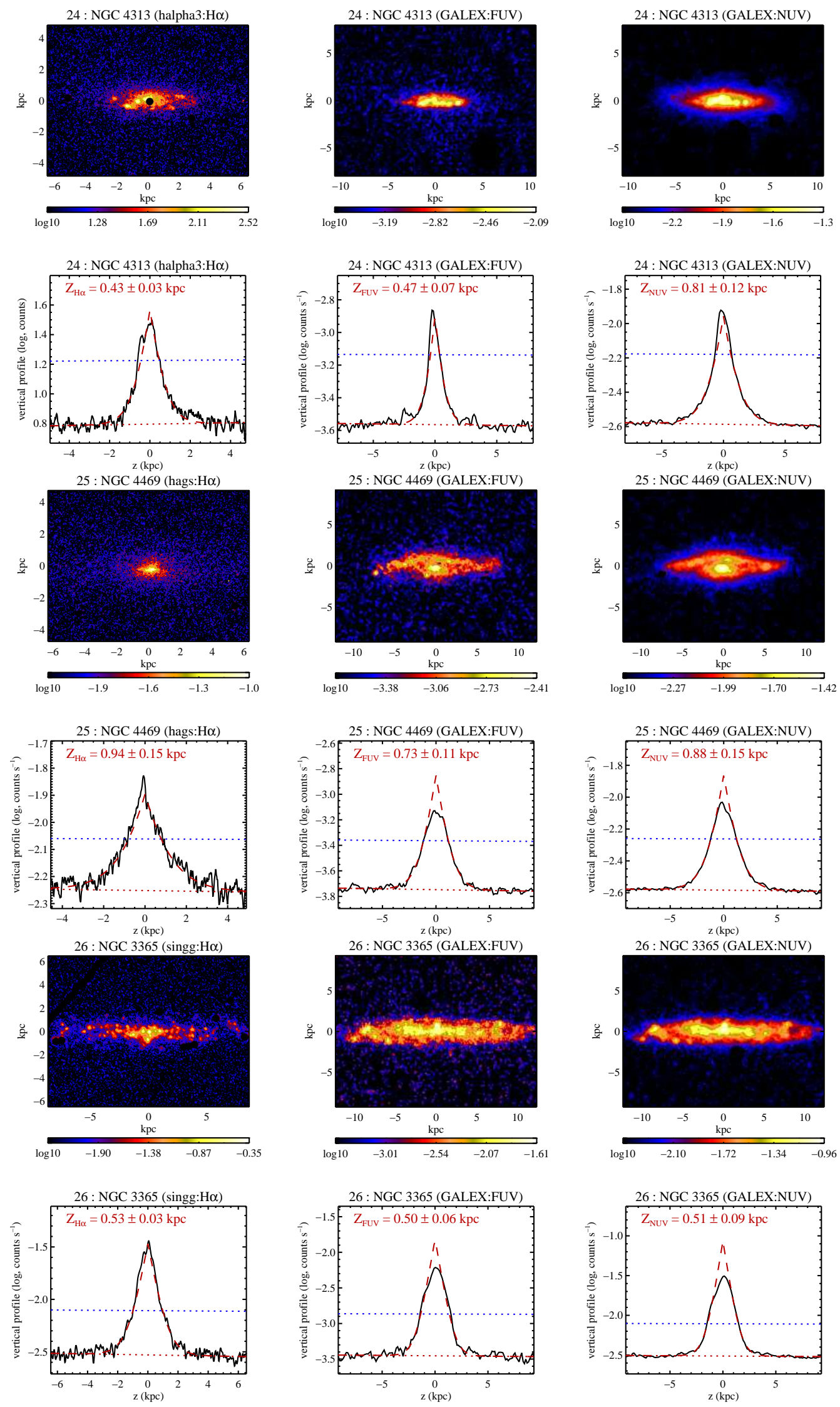

Figure 1. Continued. 

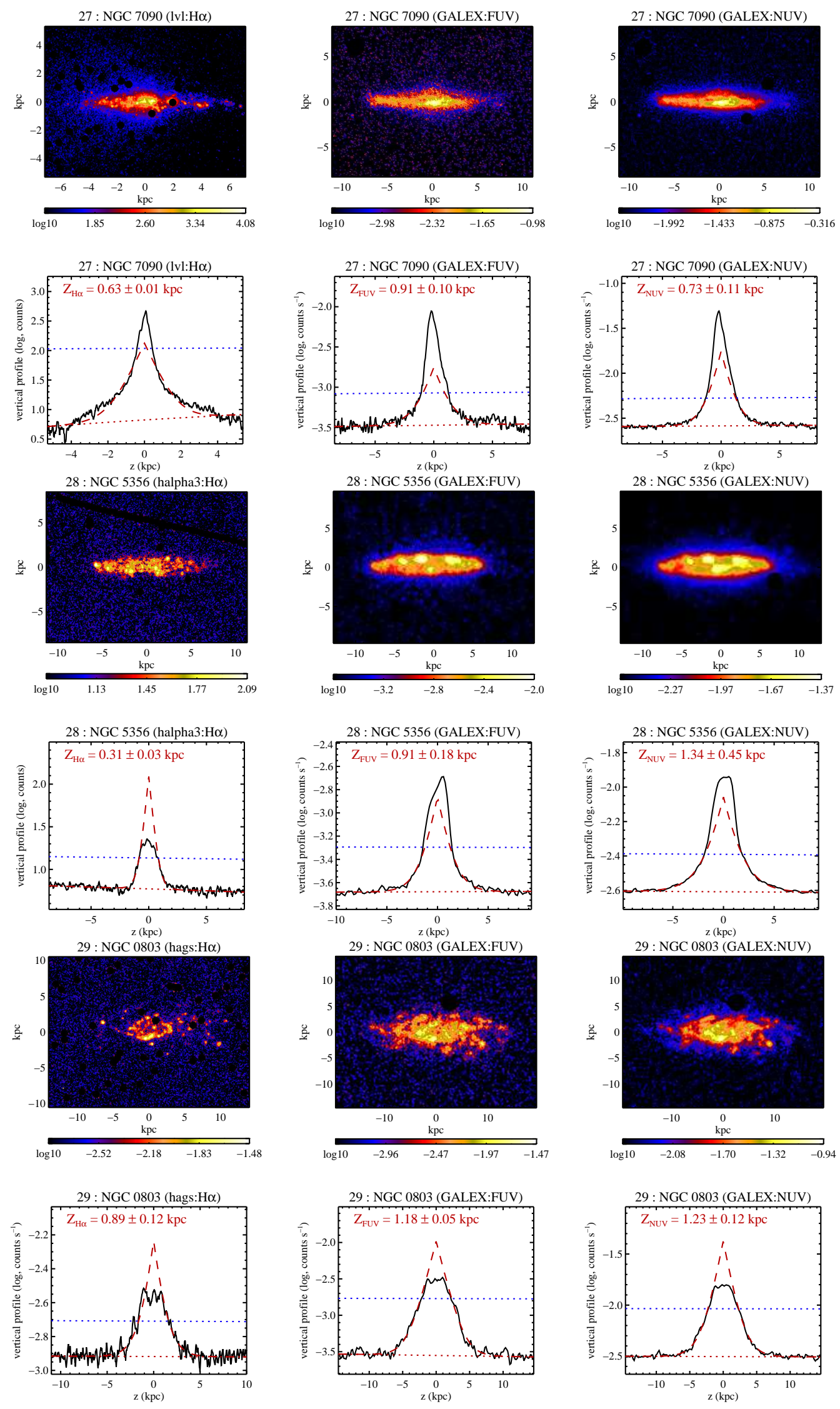

Figure 1. Continued. 

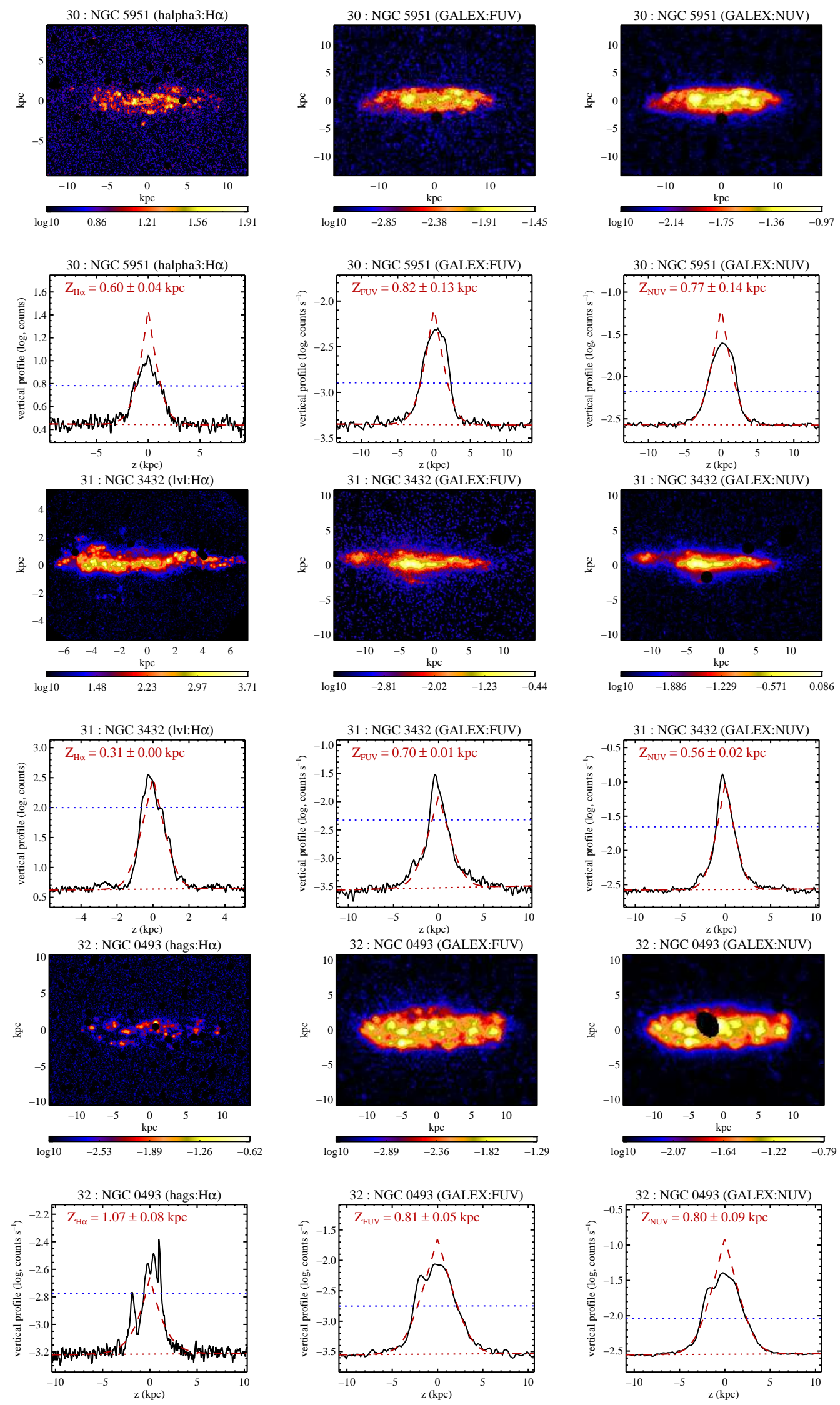

Figure 1. Continued. 

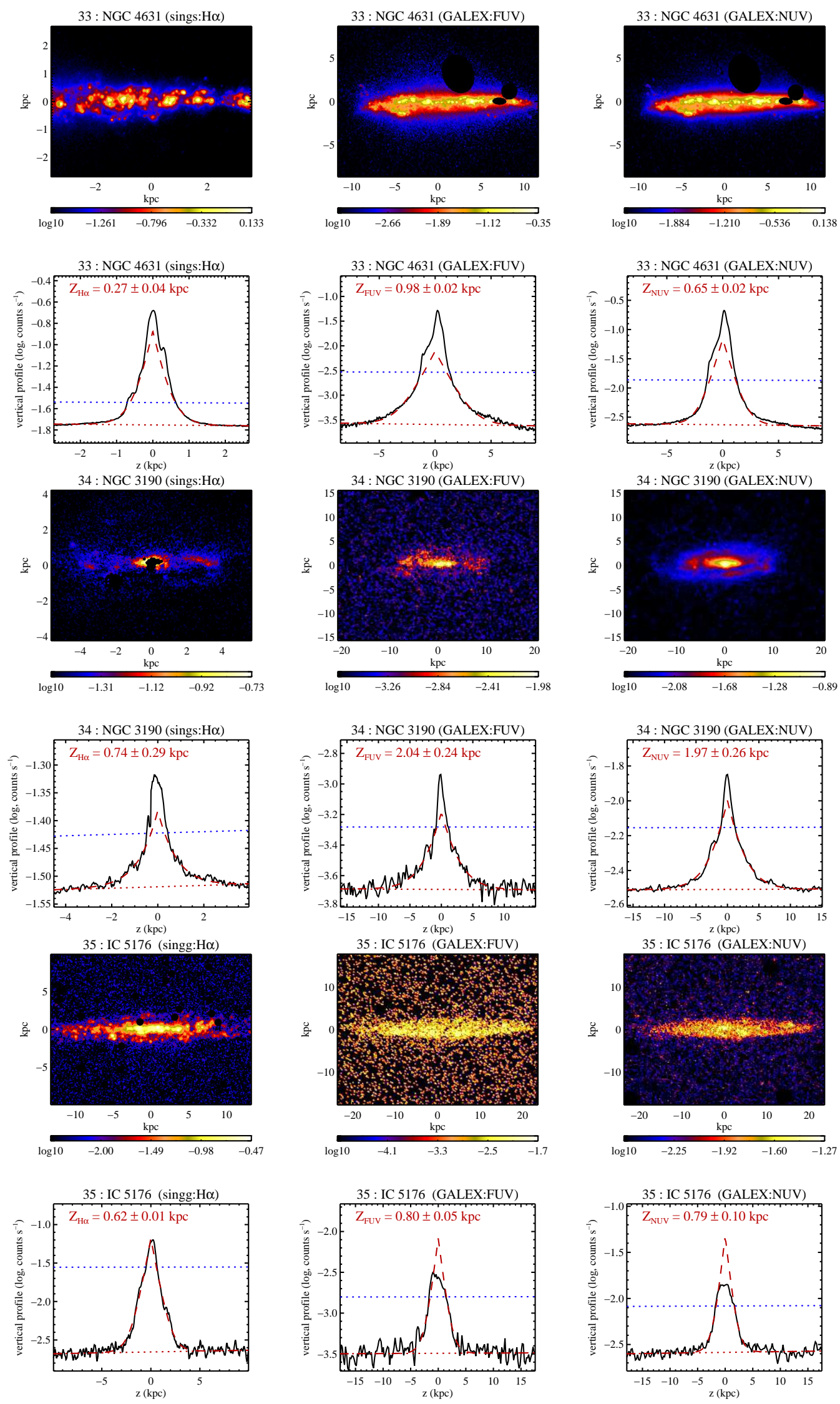

Figure 1. Continued. 

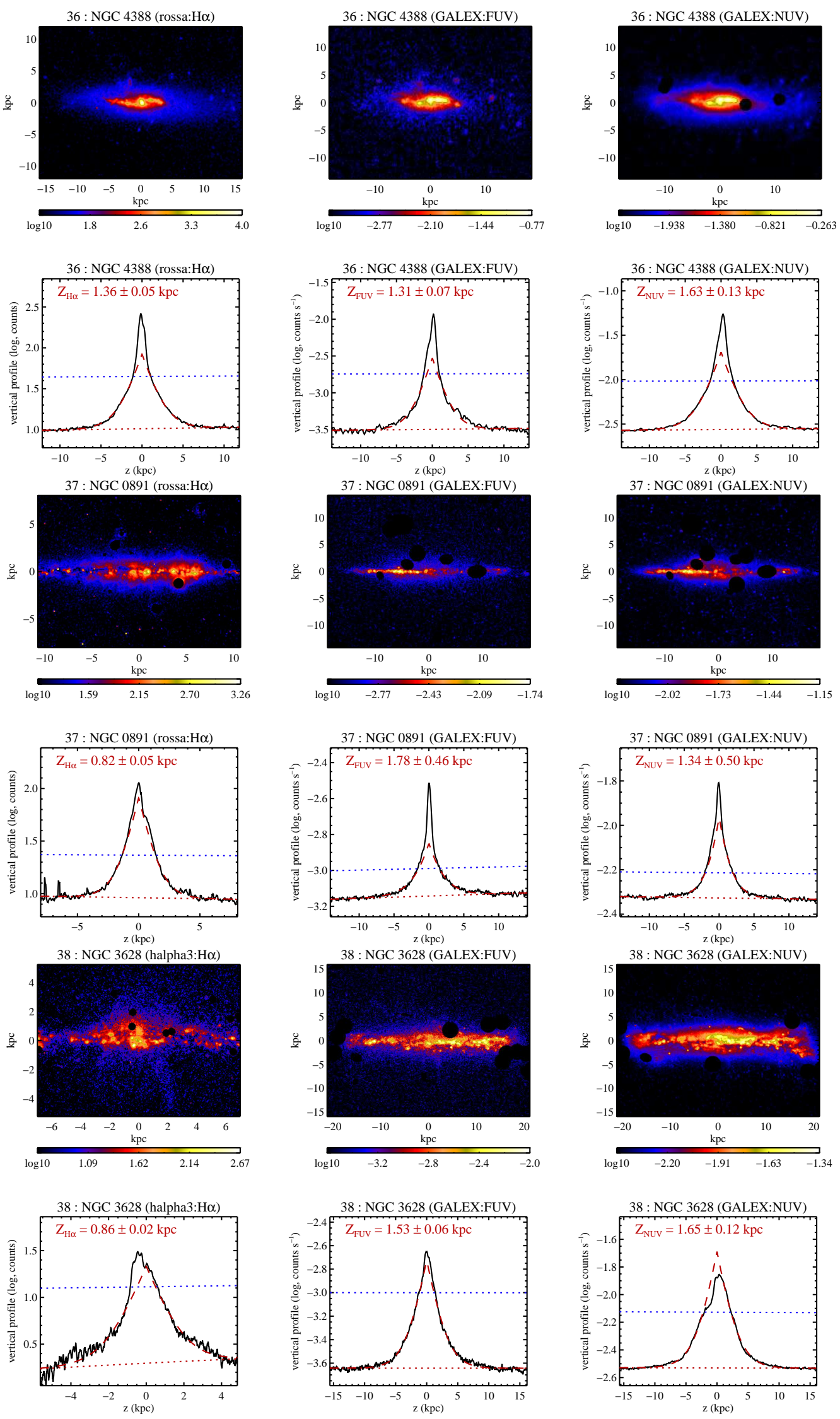

Figure 1. Continued. 
(a)

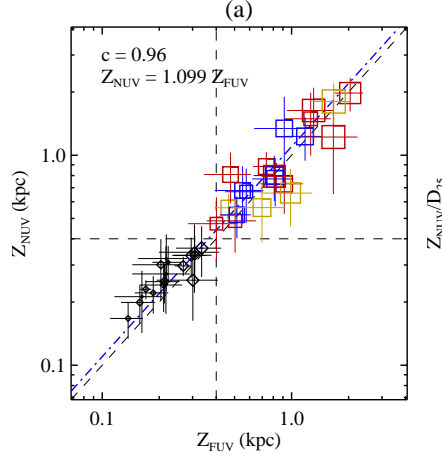

(c)

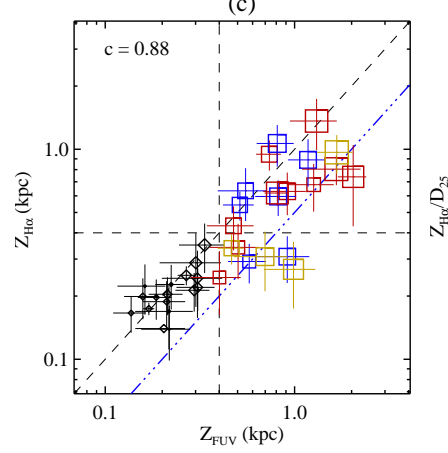

(b)

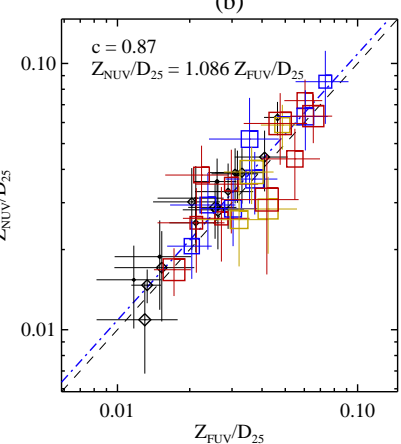

(d)

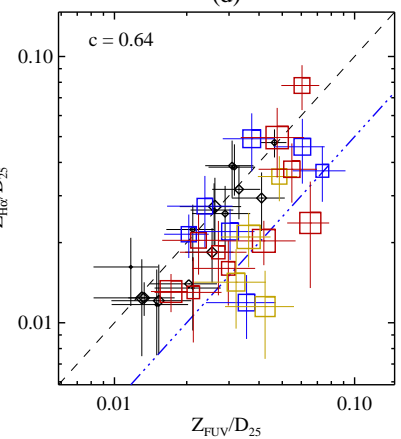

Figure 2. Comparison of the scale heights of the H $\alpha$, FUV, and NUV emissions for the 38 galaxies. Comparison of (a) scale heights of the FUV and NUV emissions in kpc, (b) relative scale heights normalized by $\mathrm{D}_{25}$ of the host galaxies for the FUV and NUV emissions, (c) scale heights measured at FUV and $\mathrm{H} \alpha$ in $\mathrm{kpc}$, and (d) relative scale heights normalized by $\mathrm{D}_{25}$ of the galaxies for the FUV and $\mathrm{H} \alpha$ emissions. Black diamonds denote Group A. Red, blue, and yellow squares denote Group B. Seven galaxies with a relatively small inclination angle (NGC 5951, NGC 493, NGC 803, NGC 4020, NGC 3365, IC 2000, and NGC 5356) and four galaxies showing disturbed disks (NGC 5107, NGC 4631, NGC 3432, and NGC 3628) are denoted by the blue and yellow squares, respectively. The remaing galaxies in Group B is denoted by the red squares. The symbol size indicates the logarithm of the galaxy size. The blue, triple-dot dashed lines in (c) and (d) denote a line corresponding to $Z_{\mathrm{H} \alpha}=0.5 Z_{\mathrm{FUV}}$.
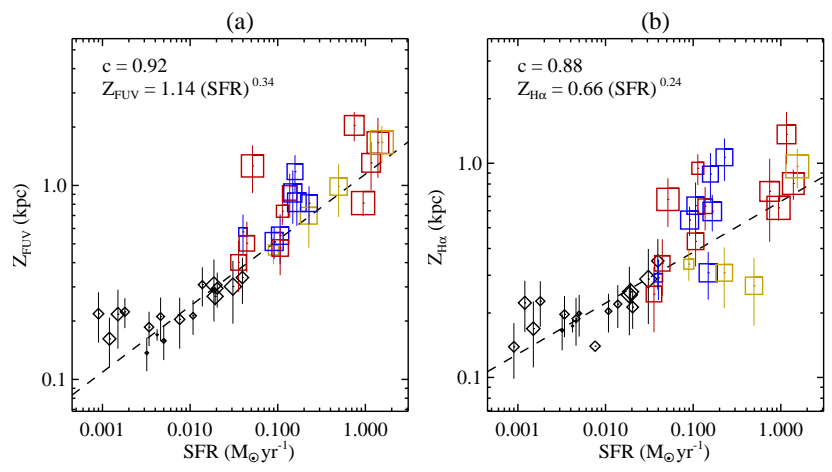

Figure 3. Comparison of the scale heights of the FUV and $\mathrm{H} \alpha$ emissions with the star formation rates (SFR FIR) of the sample galaxies. The size of the symbol is proportional to the logarithm of the galaxy size $\left(\mathrm{D}_{25}\right)$. 

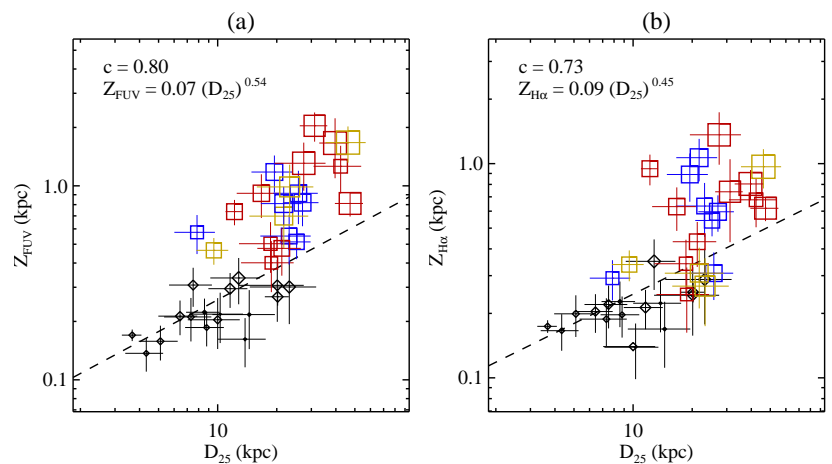

Figure 4. Comparison of the scale heights of the FUV and $\mathrm{H} \alpha$ emissions with the size of host galaxy $\left(\mathrm{D}_{25}\right)$. The size of the symbol is proportional to the logarithmic scale of star formation rates of the host galaxies $\left(\mathrm{SFR}_{\mathrm{FIR}}\right)$.

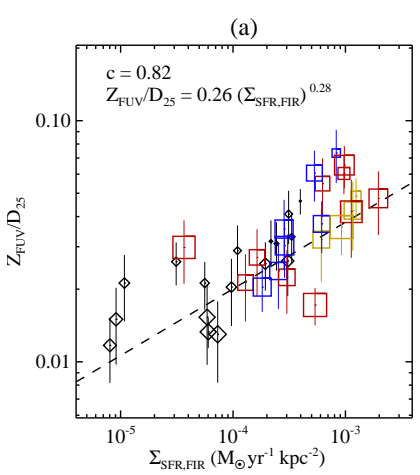

(c)

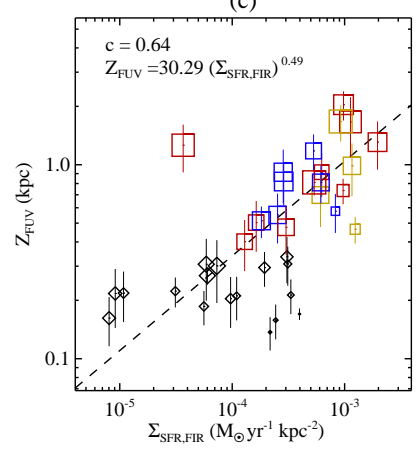

(b)

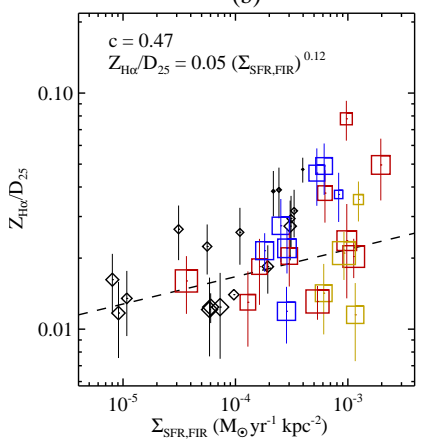

(d)

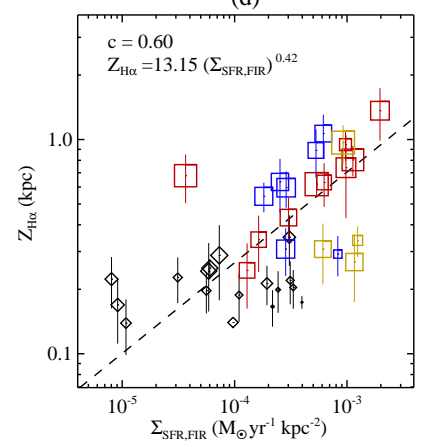

Figure 5. Comparison of the normalized scale heights of the (a) FUV and (b) H $\alpha$ emissions with star formation rate surface densities

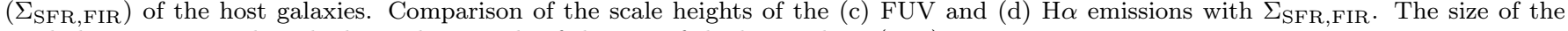
symbol is proportional to the logarithmic scale of the size of the host galaxy $\left(D_{25}\right)$.

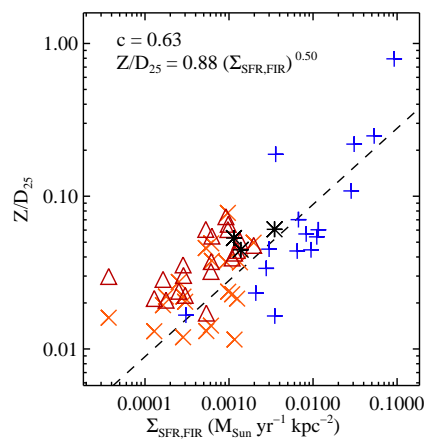

Figure 6. Comparison of the normalized scale heights of the extraplanar emissions with star formation rate surface densities $\left(\Sigma_{\mathrm{SFR}, \mathrm{FIR}}\right)$. The blue pluses indicate 16 galaxies of McCormick et al. (2013) and the black asterisks denote three galaxies of Shinn \& Seon (2015). The red triangles and orange crosses indicate the results obtained from the extraplanar FUV and H $\alpha$ emissions, respectively, of 21 galaxies (Group B galaxies) in this study. 